6

7

8

9

10

11

12

\title{
Oxytocin modulates social value representations in the amygdala
}

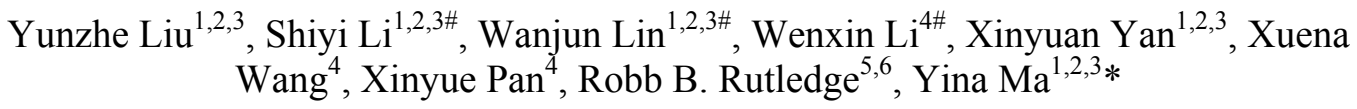
Wang ${ }^{4}$, Xinyue Pan ${ }^{4}$, Robb B. Rutledge ${ }^{5,6}$, Yina $\mathrm{Ma}^{1,2,3 *}$

1. State Key Laboratory of Cognitive Neuroscience and Learning Beijing Normal University, Beijing, China

2. IDG/McGovern Institute for Brain Research, Beijing Normal University, Beijing, China

3. Beijing Key Laboratory of Brain Imaging and Connectomics, Beijing Normal University, Beijing, China

4. School of Psychological and Cognitive Sciences, Peking University, Beijing, China

5. Wellcome Centre for Human Neuroimaging, University College London, London, UK

6. Max Planck University College London Centre for Computational Psychiatry and Ageing Research, University College London, London, UK

${ }^{\#}$ These authors contributed equally to this study

* Correspondence should be addressed to Yina Ma, Ph.D.

State Key Laboratory of Cognitive Neuroscience and Learning, Beijing Normal University, 19 Xin Jie Kou Wai Da Jie, Beijing, 100875, China

Phone/Fax: 8610-5880-2846

Email: yma@bnu.edu.cn 
33

34

35

36 Humans exhibit considerable variation in how they value their own interest relative to

37 the interests of others. Deciphering the neural codes representing potential rewards for 38

\section{ABSTRACT [149 words]} self and others is crucial for understanding social decision-making. Here we integrate computational modeling with fMRI to investigate the neural representation of social value and the modulation by oxytocin, a nine-amino acid neuropeptide, in participants evaluating monetary allocations to self and other (self-other allocations). We found that an individual's preferred self-other allocation serves as a reference-point for computing the value of potential self-other allocations. In more-prosocial participants, amygdala activity encoded a social-value-distance signal, i.e. the value dissimilarity between potential and preferred allocations. Intranasal oxytocin administration amplified this amygdala representation and increased prosocial behavior in more-individualist participants but not in more-prosocial ones. Our results reveal a neurocomputational mechanism underlying social-value representations and suggest that oxytocin may promote prosociality by modulating social-value representations in the amygdala. 
54 Humans live in complex social environments and rely heavily on social reciprocity.

55 Many of our important decisions are made in social contexts where the costs and 56 benefits to both ourselves and other people need to be considered ${ }^{1}$. Deciphering the

57 neural codes that represent potential rewards to oneself and others is crucial for 58 understanding social reciprocity and social decisions ${ }^{2}$. Recent studies of social 59 decision-making find that people are rarely purely self-centered or altruistic: they care 60 about both themselves and others' interests, but with considerable individual variation 61 in how they weigh equity of self-other gain ${ }^{3}$ and cooperation with others ${ }^{3,4}$ during their decision-making. Individuals with prosocial preferences tend to prefer allocations considering the interests of both self and other and often seek to minimize the self-other difference (henceforth prosocials). In contrast, individuals with selfish preferences tend to maximize resources for themselves and generally prefer self-centered allocations (henceforth individualists).

Individual differences in social preference may stem from individual variation in preferred social allocations and differences in neural representations of potential relative to preferred allocations ${ }^{5-7}$. It remains unclear how the difference between potential and preferred self-other allocations is computed and represented in the brain and how these computations and neural representations are related to social decision-making. Here, we propose that the preferred self-other allocation (i.e., what an individual hopes the allocation will be) serves as a social reference-point against which potential allocations are represented and that quantity can guide social value-based decisions (social reference model). The deviation from the preferred allocation generates an "error" signal that could drive adaptive actions to reduce the size of the deviation. In much the same way as reward prediction errors ${ }^{8}$ represent differences between expected and actual rewards and provide a basis for value-based decisions, ${ }^{9,10}$, this social error signal could represent deviation from the preferred allocation and serve as a basis for value-based decisions in the social domain.

The amygdala, with a large number of oxytocin and dopamine receptors ${ }^{11,12}$ and strongly implicated in social cognition and social decision-making ${ }^{3,13}$, is a prominent candidate to encode deviations from a social reference-point. Recent studies have 
84 shown that amygdala activity tracks the subjective values of rewards and

85 punishments ${ }^{14}$ and reflects individual preferences ${ }^{15}$. Notably, the amygdala has been

86 suggested to encode error signals that represent the differences between expectations

87 and outcomes ${ }^{9}$, a quantity that is fundamental for value-based decision-making ${ }^{2}$.

88 Amygdala activity has also been shown to encode "aversive" signals to absolute

89 inequality when evaluating reward pairs for self and other ${ }^{3}$ and in response to dishonest

90 behavior $^{16}$. One untested possibility is that amygdala activity encodes the deviation of a

91 potential self-other allocation from a reference-point that depends on

92 individual-specific social preferences.

93 Prosocials and individualists, who should differ in their social reference-point, would

94 be expected to engage different neural substrates for social value representations. For

95 prosocials, the distance from their social reference-point could signal deviation from

96 normative social principles such as inequity aversion, which is associated with the

97 amygdala ${ }^{3}$. On the other hand, individualists employing a self-interest maximizing

98 strategy in their decision-making could represent deviation from this reference-point

99 mainly as conflict with self-interest, engaging lateral prefrontal regions associated with

100 inhibition of self-interest and self-other allocation trade-off ${ }^{17}$, such as lateral

101 orbitofrontal cortex (lOFC) ${ }^{18,19}$ and dorsolateral prefrontal cortex (dlPFC) ${ }^{20}$.

102 It has also been suggested that individual differences in social behavior result in part

103 from differences in the neuromodulatory regulation of neural circuits ${ }^{6}$. The

104 neuropeptide oxytocin, an evolutionarily conserved hormone, is a potential candidate ${ }^{21}$.

105 Oxytocin has been found to play an important role in social interaction and social

106 decision-making ${ }^{22}$, including promoting social motivation ${ }^{23}$, increasing trust and

107 cooperation with own-group members ${ }^{24}$, and reducing social distance ${ }^{25}$. Whether and

108 how oxytocin modulates the basic computations of social preference and social value

109 representations remains largely unexplored. Individual differences in social

110 preferences in nonhuman primates have been shown to be due in part to oxytocinergic

111 regulation of amygdala-related neural circuits ${ }^{6,7}$. In nonhuman primates, exogenous

112 inhaled oxytocin promotes social donation behavior ${ }^{26}$ and focal infusion of oxytocin

113 into the amygdala significantly increases prosocial decisions ${ }^{7}$. In humans, it has been

114 suggested that individual differences in oxytocin effects are adaptive depending on an 
115 individual's social disposition ${ }^{21}$ such that intranasal oxytocin produces stronger effects

116 on cooperation in less socially proficient individuals ${ }^{4}$. Oxytocin differentially impacts

117 cooperative and aggressive choices in individuals with different pre-existing beliefs in

118 prosociality $^{27}$. We therefore predicted differential effects of oxytocin on regulating

119 prosocial behavior between prosocials and individualists by selectively increasing

120 prosociality in individualists via amplification of amygdala social value

121 representations.

122 Here, we set out to test whether intranasal administration of oxytocin differentially

123 modulates the neural representation of social values in prosocials and individualists

124 performing a monetary outcome-pair evaluation task during fMRI scanning in a

125 double-blind placebo-controlled between-subjects design. We first show that our social

126 reference model most parsimoniously explains behavior consistent with social values

127 being encoded as distance to an individual-specific reference-point. While prosocials

128 represent social values relative to a more prosocial reference-point than individualists,

129 oxytocin selectively increased prosociality in individualists and not prosocials in both

130 competitive and non-competitive contexts. Moreover, these findings were replicated in

131 two additional behavioral experiments. Using model-based fMRI analysis, we found

132 that under placebo, amygdala activity in prosocials encodes a social value distance

133 reflecting the degree to which a potential self-other allocation deviates from an

134 individual-specific reference-point. Oxytocin selectively amplified the neural

135 representation of social values in the amygdala in individualists, suggesting a link

136 between oxytocin and prosociality via modulation of social value representations in the 137 amygdala.

\section{RESULTS}

\section{Experimental settings}

140 In the fMRI experiment, we first invited participants $(n=282)$ to a behavioral session to

141 identify their social dispositions (i.e., prosocials vs. individualists) using the triple

142 dominance ${ }^{5}$ and social value orientation $(\mathrm{SVO})^{28}$ decision-making tasks

143 (Supplementary Fig. 1). Thereafter, eligible prosocials and individualists $(n=127)$

144 administered oxytocin or placebo performed a monetary outcome-pair evaluation task 
145 during fMRI scanning (Fig. 1a). On each trial, participants were presented with pairs of

146 monetary outcomes for himself and another participant (referred to as the partner) and

147 evaluated his preference on each pair.

148 All monetary allocations were evenly sampled on the circumference of a circle centered

149 at the origin $(0,0)$ in the Cartesian coordinate space spanned by social values (with

150 monetary outcomes for self as the x-axis and outcomes for the partner as the y-axis,

151 radius $=5$, Fig. 1a). Monetary outcomes for oneself and the partner define an angle $\theta$,

152 which samples the space from $-90^{\circ}$ to $180^{\circ}$. The angle between any two potential

153 allocations is both necessary and sufficient to quantify their relationship. Both positive

154 and negative values were included for a comprehensive investigation of social value

155 representations, except for those in the third quadrant due to invariant preference rating

156 shown in an independent sample (Methods).

157 We also ran two additional behavioral experiments, one experiment with a large sample

158 (behavioral online-replication experiment, $n=315$ ) providing a replication for our

159 finding that the social reference model outperforms other models and one experiment

160 providing a replication of the oxytocin effect (oxytocin-replication experiment, $n=80$

161 males, within-subjects design, 40 prosocials and 40 individualists). To improve the

162 ability to distinguish between different models, both additional experiments were run

163 on a modified design where monetary pairs were sampled on 3 circles of different

164 circumference (radius $=5,6,9$ ), with $\theta$ ranging from $-90^{\circ}$ to $180^{\circ}$ with different

165 intervals $\left(5^{\circ}, 17^{\circ}, 23^{\circ}\right)$. These specifications were identified based on model recovery

166 analysis, which suggested that the combination of these parameters would lead to

167 maximal discriminability between our social reference model and an inequality

168 aversion model. The task design for these additional experiments was otherwise

169 identical to the fMRI experiment.

\section{Representing social values according to an individual-specific reference-point}

171 In the fMRI experiment, we first plotted z-scored preference ratings for each social

172 allocation across participants for visualization purposes (Fig. 1b). In general,

173 participants most preferred self-gain/other-gain pairs and least preferred

174 self-loss/other-gain pairs, suggesting that participants considered the interests of both

175 self and partner. Based on preference ratings for all allocations, we computed an

$$
6 / 37
$$


176 individual-specific reference-point, referred to as $\varphi$. The principle of $\varphi$ calculation was 177 consistent with the "mean orientation" measure in a map-like structure ${ }^{29,30}$ (Fig. 1c).

178 The degree of $\varphi$ indicated how much a participant preferred the potential outcome for

179 the partner in relation to himself, with larger angles corresponding to stronger

180 preference for allocations that benefit the partner relative to oneself and thus greater

181 prosociality.

182 We then calculated cosine similarity between each allocation $\theta$ and the

183 individual-specific reference-point $\varphi$ to compute social value distance, the dissimilarity

184 distance of that allocation to the participant's preferred allocation (also the deviation

185 from the social reference-point, calculated as $1-\operatorname{cosine}(\theta-\varphi))$. This measurement

186 allowed us to quantify the difference between the second (self-loss/other-gain pairs)

187 and fourth (self-gain/other-loss pairs) quadrants (Fig. 1c), which is not feasible when

188 only including gains or using absolute value differences ${ }^{3,28}$. The social reference model

189 was consistently the most parsimonious model across all studies: the fMRI during-scan

190 experiment, the post-scan behavioral experiment, the behavioral online-replication

191 experiment and the oxytocin-replication experiment (supported by model comparisons

192 using variational free energy as the model selection criteria, Supplementary Fig. 2).

193 More prosocial reference-points for social value representations in prosocials

194 We quantified the difference in the estimated reference-point between prosocials and 195 individualists under placebo. We found significantly higher values of $\varphi$ in prosocials 196 than individualists $\left(F(1,59)=33.49, p=2.91 \times 10^{-7}, \eta^{2}=0.36\right.$, Fig. 2a), which was

197 replicated in the large sample online experiment $\left(F(1,313)=92.14, p=2.71 \times 10^{-19}\right.$,

$198 \eta^{2}=0.23$, Fig. 2b). Moreover, in the online-replication experiment, the social

199 reference-point derived from the social reference model was correlated with

200 individuals' SVO scores $\left(r=0.55, p=4.38 \times 10^{-26}, 95 \% \mathrm{CI}=[0.47,0.62]\right.$, Fig. 2c),

201 suggesting a more prosocial reference-point in prosocials both at a group and individual

202 level. The pattern of more prosocial reference-points in prosocials than individualists

203 was similarly observed in the competitive context in the post-scan experiment

$204\left(F(1,59)=12.59, p=7.69 \times 10^{-4}, \eta^{2}=0.18\right.$, Fig. 2d $)$ and in the oxytocin-replication

205 experiment $\left(F(1,78)=28.27, p=9.78 \times 10^{-7}, \eta^{2}=0.27\right.$, Fig. 2e). 
207 Under placebo, $\varphi$ was significantly correlated with independent measures of previously

208 established prosocial behavior (Methods), with positive correlations between $\varphi$ and the

209 amount of contribution in a public goods game $\left(r=0.52, p=2.44 \times 10^{-5}\right)$ and in a dictator

210 game $(r=0.44, p=0.0006)$. The degree of $\varphi$ was also correlated with the degree of

211 absolute inequality aversion (which reflected a general preference for fairness and

212 resistance to inequalities, with higher values indicating higher inequality aversion,

$\left.213 r=0.65, p=1.54 \times 10^{-8}\right)$, measured in an independent $\operatorname{task}^{28}$ (Methods). Note that a $\varphi$ of $45^{\circ}$

214 indicates a preference for equal offers. Within a fairly small range of $\varphi$ corresponding

215 to most of our participants $\left(-30\right.$ to $\left.45^{\circ}\right)$, the larger the $\varphi$, the more prosocial a

216 participant.

217 It has been suggested that decision time reflects perceived conflicts between prior

218 expectation and current choice, with faster responses for preferred and less conflicted

219 choices $^{31,32}$. Thus, we would expect faster decision-making when conflict is minimal

220 and the potential allocation is close to the individual-specific reference-point. As the

221 social value distance increased, longer decision times were predicted. We indeed found

222 that decision time for a potential allocation increased as a function of deviation from

223 individual-specific reference-point, and such correlation was stronger in individualists

224 than prosocials under placebo (independent-samples t-test on the Fisher z-scored

225 correlation coefficients, individualists vs. prosocials: $0.18 \pm 0.03$ vs. $0.09 \pm 0.02$;

$226 \mathrm{t}(59)=2.33, \mathrm{p}=0.023$ in the fMRI experiment, with a similar trend in the

227 oxytocin-replication experiment, paired-samples t-test: $\mathrm{t}(78)=1.86, \mathrm{p}=0.067$,

228 Supplementary Fig. 3). The greater the dissimilarity between potential and preferred

229 allocations, the longer individualists took to evaluate potential allocations.

\section{Selective oxytocin effects on promoting prosociality in individualists}

231 We evaluated the oxytocin effect on the social value representations. First, we checked

232 the relationship between baseline salivary oxytocin and social value representations

233 across all participants. The individual-specific reference-point $\varphi$ was independent of

234 baseline salivary oxytocin $(r=0.03, p=0.75)$ (Supplementary Fig. 4 ). We also measured

235 participants' social perceptions of their partner by rating the first impression, likeability 
236 and attractiveness of the partner. There was no significant difference across all groups 237 on any of these measures (Supplementary Fig. 5). Therefore, any significant effect of

238 Social Disposition and/or Treatment on the social value representation cannot be 239 attributed to baseline oxytocin or social perception differences.

240 We conducted ANOVA on $\varphi$, with Social Disposition (prosocial vs. individualistic) 241 and Treatment (oxytocin vs. placebo) as between-subjects factors. There was a 242 significant main effect of Social Disposition $\left(F(1,121)=28.09, p=5.29 \times 10^{-7}, \eta^{2}=0.19\right)$, 243 with prosocials (vs. individualists) using a more prosocial reference-point to evaluate 244 potential allocations. Interestingly, we found a significant Social Disposition $\mathrm{x}$ 245 Treatment interaction $\left(F(1,121)=6.35, p=0.013, \eta^{2}=0.05\right.$, Fig. 2a), as intranasal 246 oxytocin significantly increased the reference-point $\varphi$ towards a preference for more 247 prosocial allocations in individualists (independent-samples $t$-test, $t(57)=2.21$, $248 p=0.031)$, but not in prosocials $(t(64)=-1.54, p=0.13$, Fig. 2a), indicating that oxytocin 249 selectively increased prosociality in individualists. Furthermore, there was no effect of 250 scanning order or partner type on $\varphi$ (Supplementary Fig. 6).

251 We replicated the selective oxytocin effect on promoting prosociality in individualists 252 in the independent oxytocin behavioral experiment where we employed a 253 within-subjects design and included monetary pairs sampled on 3 circles of different 254 circumferences (Social Disposition: $\mathrm{F}(1,78)=19.51, p=3.19 \times 10^{-5}, \eta^{2}=0.20$; Social 255 Disposition $x$ Treatment interaction: $F(1,78)=6.73, p=0.011, \eta^{2}=0.079$, Fig. 2e).

256 Moreover, the within-subjects design, where each participant was invited to both 257 oxytocin and placebo sessions, allowed us to examine whether the oxytocin effect 258 varied as a function of individual scores in social value orientation. We expected a 259 negative correlation between SVO scores and the oxytocin effect on prosociality, and 260 indeed found a significant negative correlation between SVO scores and the size of 261 oxytocin effect on social reference-point ( $r=-0.23, p=0.041$, Fig. 2f), suggesting that 262 the more selfish the individual, the stronger the effect of oxytocin on promoting a 263 prosocial reference-point.

264 Finally, to determine whether the lack of oxytocin effect on prosocials was due to a 265 ceiling effect (i.e., prosocials already care about others' outcomes), we introduced a 266 competitive social context in the post-scan behavioral task where self-interest and 267 other-interest were in direct competition. In the competitive context, we framed the 
payoff in a "winner takes all" manner, so that one would be motivated to make selfish decisions to gain more than the partner. If oxytocin can promote prosociality in prosocials, which is masked by a ceiling effect in the non-competitive setting, we would expect oxytocin to affect prosocials in the competitive context. We conducted an ANOVA on $\varphi$ in prosocials, with Treatment as between-subjects factor and Context (competitive vs. non-competitive) as within-subjects factor. There was a significant main effect of Context $\left(F(1,64)=68.61, p=1.02 \times 10^{-11}, \eta^{2}=0.52\right)$, but no Treatment effect $(F(1,64)=0.856, p=0.358)$, or interaction with Context $(F(1,64)=1.33, p=0.254)$, suggesting that the lack of a prosocial effect of oxytocin in prosocials was not due to a ceiling effect given their relative "self-centered" social value preference in the competitive compared to non-competitive context. A similar ANOVA on individualists showed that oxytocin increased prosociality for individualists across both competitive and non-competitive contexts (Treatment: $\left(F(1,57)=8.56, p=0.005, \eta^{2}=0.131\right.$; Treatment $x$ Context: $F(1,57)=0.018, p=0.894)$. Furthermore, the ANOVA on $\varphi($ Fig. 2a, d), with Social Disposition and Treatment as between-subjects factors and Context as a within-subjects factor, revealed the expected significant main effect of Context $\left(F(1,121)=145.92, p=1.59 \times 10^{-22}, \eta^{2}=0.55\right)$, with decreased prosociality in the competitive context. There was a significant Social Disposition $\mathrm{x}$ Treatment interaction $\left(F(1,121)=5.28, p=0.023, \eta^{2}=0.042\right)$. Moreover, this interaction was not affected by Context $(F(1,121)=0.697, p=0.406)$, suggesting that the oxytocin effect on promoting prosociality was selective to individualists across multiple contexts.

\section{Amygdala in prosocials represents a social value distance signal}

Based on the behavioral model, we looked for brain regions that encoded the social value distance between potential and preferred allocations. We created a parametric modulator for the social value distance $\mathrm{e}^{29,30}: 1-\cos (\theta(\mathrm{t})-\varphi)$, based on our social reference model, where $\theta(\mathrm{t})$ is the angle of a current allocation at trial $\mathrm{t}$ and $\varphi$ is the individual-specific reference-point. This measure reflects the degree to which an allocation deviates from the reference-point, with higher values indicating greater distance (i.e., lower desirability) between potential and preferred allocations. At the second-level analysis, we found that the posterior cingulate cortex (peak coordinate in MNI space: -6/-64/42) and middle frontal gyrus (peak coordinate in MNI space: $-34 / 8 / 54$ ) encoded social value distance when collapsing over the four groups 
300 (whole-brain significant at a voxel-wise threshold $\mathrm{p}<0.001$ and a cluster-wise FWE correction with $\mathrm{p}<0.05$ ).

302 We then searched for brain regions that encoded the social value distance respectively

303 for individualists and prosocials. We found that, under placebo, amygdala activity

304 encoding social value distance was significantly stronger in prosocials than

305 individualists (voxel-wise threshold $\mathrm{p}<0.001$ and a cluster-wise FWE correction with

$306 p<0.05$, peak MNI coordinate: 20/-10/-12, Fig. 3a, Supplementary Table 1). Previous

307 studies have linked inhibition of self-interest and top-down control of selfish behavior

308 with right lateral prefrontal cortex ${ }^{17}$, such as right $1 \mathrm{OFC}^{18,19}$ and right $\mathrm{dlPFC}^{20}$. We

309 hypothesized that individualists would represent deviation from preferred allocations

310 mainly as a conflict with self-interest and a social value distance representation might

311 be present in these areas. Comparison of individualists and prosocials under placebo

312 revealed that right $1 \mathrm{OFC}$ activity encoded social value distance to a greater degree in

313 individualists than prosocials (voxel-wise threshold $\mathrm{p}<0.001$, small volume correction

$314 \mathrm{p}<0.05$ for an anatomically defined right 1OFC mask, using combined

315 connectivity-based parcellations $8-11$ covering right $1 \mathrm{OFC}^{33}$, Fig. $\left.3 \mathbf{b}\right)$. This

316 relationship was not present for right dIPFC. Further region of interest (ROI) analysis

317 revealed a significant interaction between brain areas (right 1OFC vs. amygdala) and

318 Social Disposition (individualist vs. prosocials) under placebo $(F(1,58)=11.210$,

$319 p=0.0014, \eta^{2}=0.162$ ). Here, the amygdala and right lOFC ROIs employed anatomically

320 defined masks.

321 We extracted beta estimates associated with encoding social value distance from an

322 anatomically defined amygdala ROI. We found that the strength of the amygdala social

323 value distance representation was correlated with the degree of inequality aversion

324 ( $r=0.296, p=0.0012$, Supplementary Fig. 7), whereas right 1OFC activity bore no

325 relationship to inequality aversion $(r=-0.13, p=0.59)$. A moderation analysis revealed

326 that this positive correlation was significantly stronger in prosocials than individualists

327 under placebo $\left(\mathrm{R}^{2}\right.$ change $=0.06, p=0.003$, Supplementary Fig. 7).

328 Oxytocin modulates social value representations in the amygdala in individualists 
We then searched for the main effect of Treatment and the interaction effect of Social Disposition and Treatment in the whole brain. There was no significant main effect of Treatment. The Social Disposition $\mathrm{x}$ Treatment interaction $F$ contrast revealed a significant cluster in the amygdala (Fig. 4a, peak voxels in the right amygdala survived voxel-wise FWE correction: $p<0.05)$. Intranasal oxytocin selectively amplified the neural representation of social value distance in the amygdala of individualists, but not prosocials. A similar interaction pattern was found in other brain regions, including the right temporoparietal junction (TPJ) and ventral striatum (Supplementary Fig. 8). Moreover, as illustrated in Supplementary Fig. 9a-d, amygdala activity increased as a function of deviation from an individual-specific reference-point in prosocials under placebo (slope estimate of the linear fit $=0.222, p=0.001$ ) and this pattern was not found under oxytocin (slope estimate $=0.010, p=0.88$ ). In contrast, amygdala activity increased as a function of deviation from an individual-specific reference-point in individualists under oxytocin (slope estimate $=0.232, p=0.003$ ) and this pattern was not found under placebo (slope estimate $=0.042, p=0.50$ ). Amygdala responses were not related to absolute value differences or deviations from the allocentric reference (Supplementary Fig. 9e-l, all p>0.5).

We then examined the relationship between neural responses and evaluations of monetary allocations on a trial-by-trial basis to test whether the amygdala or right 1OFC activity explained trial-by-trial variation in subjective preference ratings that was independent of the predicted social value distance. At the first-level (individual-subject-level) analysis, we modelled each trial separately and extracted beta estimates for amygdala and right $1 \mathrm{OFC}$ for each trial. We then regressed the trial-by-trial amygdala and right $1 \mathrm{OFC}$ responses (as $\mathrm{x}$ in the regression) respectively onto the evaluation made for each monetary allocation on each trial (as $y$ in the regression), while controlling for the deviation of each potential allocation from the individual-specific reference-point. In doing so, we ensure that the beta estimates associated with the trial-by-trial amygdala and right $1 \mathrm{OFC}$ activity reflect unique 357 variance in predicting preference ratings based on amygdala and right $1 \mathrm{OFC}$ responses 358 above and beyond variance explained by predicted social value distance. We conducted 359 a Social Disposition-by-Treatment ANOVA on the trial-by-trial correlation coefficient 360 and found a significant interaction between Social Disposition and Treatment 
362 negatively predicted trial-by-trial preference ratings under placebo, which was reduced

363 by oxytocin. In contrast, amygdala activity negatively predicted trial-by-trial

364 preference ratings in individualists under oxytocin vs. placebo. The negative

365 correlation indicated that the stronger the amygdala activity encoding social value

366 distance, the lower the preference. This pattern of results was consistent with the

367 amygdala providing an input signal for preferences, and fluctuations in the amygdala

368 responses can explain trial-by-trial deviations from average preferences. No such

369 results were found for right $1 \mathrm{OFC}$ activity.

370 Furthermore, we conducted a general linear model (GLM) with preference rating for

371 each monetary allocation as a parametric modulator to identify any neural activity

372 sensitive to subjective preference ratings. We found activity in the medial prefrontal

373 cortex (mPFC) and lOFC, brain regions typically associated with value-coding ${ }^{34,35}$,

374 correlated with subjective preference ratings for monetary allocations collapsing across

375 the four groups. Moreover, there was a significant interaction between Social

376 Disposition and Treatment for the mPFC and 1OFC activity that encoded preference

377 ratings (height threshold $p<0.001$, cluster-based FWE correction, $p<0.05$;

378 Supplementary Fig. 10). No significant Social Disposition $\mathrm{x}$ Treatment interaction was

379 found in the amygdala encoding the preference rating at the whole-brain or ROI level,

380 suggesting that the amygdala activity encoding social value distance does not simply

381 reflect the reverse of the preference signal.

382 We performed a generalized psycho-physiological interaction (gPPI) analysis with

383 anatomically defined bilateral amygdala as the seed region at the whole-brain level. We

384 found that amygdala activity encoding social value distance was coupled with ventral

385 mPFC activity. Moreover, amygdala-vmPFC coupling was significantly stronger in

386 prosocials than in individualists under placebo (height threshold $p<0.001$, uncorrected,

387 Supplementary Fig. 11). Further, the ROI analysis suggested that oxytocin increased

388 the strength of functional connectivity between amygdala and vmPFC in encoding

389 social value distance in individualists (independent-samples $t$-test, $t(54)=2.69, p=0.009$ )

390 but not in prosocials $(t(58)=0.067, p=0.95$, Supplementary Fig. 11). Given that the

391 vmPFC is typically associated with value computation and value-guided choice ${ }^{34,35}$,

392 these results may suggest a potential amygdala pathway linked to social preferences

393 and social value-based decisions, which can be modulated by oxytocin in 
individualists.

\section{DISCUSSION}

396 Our results suggest that the representation of social values is a relational map that encodes the distance between potential values for oneself and others on the same coordinate system. This representation can guide how we interact with others and how we respond to perceived unfairness. We provide empirical evidence that social value representations are constructed in relation to individual-specific social preferences, with the distance between potential and preferred allocations determining the value of social allocations. Prosocials represent social values relative to a more prosocial reference-point than individualists, even in a competitive social context where self- and other-interest are in direct competition. Moreover, the social reference-point derived from our social reference model accounts for individual variation in prosocial behaviors (e.g., cooperation, generosity and inequality aversion) and therefore could serve as a compact description of social decision-making.

408 This dissimilarity distance measure bears some similarity to variables in value-based decision-making frameworks ${ }^{2}$. Social value distance is encoded by the amygdala in prosocials: the more dissimilar a potential allocation to the individual-specific reference-point (i.e., their preferred allocation), the greater the amygdala response. Our results offer a mechanistic account of how social value representations contribute to decision-making in prosocials. Trial-by-trial amygdala activity encoding social value

414 distance reflects how attractive potential social allocations are judged to be by 415 prosocials (i.e., the stronger the amygdala response, the less attractive the allocation).

416 Our control analyses show that amygdala activity is better explained by

417 individual-specific reference-points than by egocentric or allocentric frames of 418 reference (Methods). Thus, amygdala activity might encode the difference between 419 potential and preferred allocations (i.e., a "surprise" signal) much like dopamine firing represents reward prediction errors reflecting the difference between outcomes and expectations $^{10}$.

422 We found an amygdala representation of social value distance that reflects a deviation 423 from the most preferred allocation (what an individual hopes the allocation to be). This 424 result is consistent with studies in both nonhuman primates ${ }^{7}$ and in human 
neuroimaging studies ${ }^{16}$ suggesting that the amygdala represents how undesirable an outcome is. Providing further support, we found that trial-by-trial amygdala activity was negatively correlated with the desirability of potential social allocations. Moreover, we found evidence that social value distance is also encoded by neural responses in the ventral striatum and TPJ in prosocials, consistent with previous findings linking prosocial decisions with several hubs in the social brain network ${ }^{7,36}$, including the amygdala, ventral striatum and TPJ. For example, TPJ activity encodes the subjective value of altruistic choice and the value of generosity ${ }^{36-38}$.

We also found some evidence for a social value distance representation in the right 1OFC in individualists relative to prosocials, which may reflect a distinct coding scheme for representing social values, although this activity did not survive whole-brain cluster-level correction and was not predictive of trial-by-trial preference ratings or modulated by oxytocin. However, this pattern is consistent with previous studies related to right 1 OFC function ${ }^{39}$. Right 1 OFC activation is associated with inhibition of self-interest, reward-guided decisions and detecting and evaluating threats to self-interest ${ }^{18,19}$. The right dlPFC, another region implicated in social decision-making and top-down control of selfish behavior ${ }^{20}$, did not have any significant social value distance representation in either individualists or prosocials.

Taken together, individuals may consider or calculate self-interest, altruistic values and evaluation of threat to self-interest when comparing potential and preferred allocations. These processes differ among individuals with different social orientations. Social value distance signals in prosocials may also relate to mentalizing about the needs of others $^{36,37}$, integrating social information into estimates of subjective value $e^{40}$, and calculating altruistic values ${ }^{41}$ in the social brain network. However, it is possible that individualists perceive deviations from their preferred allocation mainly as conflicts with self-interest, consistent with studies related to inhibition of self-interest conflict and evaluating threats to self-interest ${ }^{18,19}$.

Oxytocin is believed to facilitate social approach and to increase the salience of social cues in promoting adaptive social behaviors ${ }^{21}$. While individualists focus on self-interest and personal goals when making decisions ${ }^{3,4}$, oxytocin may increase prosociality by shifting reference-points to more prosocial allocations and increasing the weights of outcomes for others, possibly through amplifying the amygdala 
representation of social value distance. This is consistent with studies showing that the amygdala plays a critical role in allocating attention to other people ${ }^{7}$ and in integrating social information $^{42}$ and social emotions ${ }^{13}$ into decision-making. However, oxytocin fails to show a prosocial effect in prosocials. This does not necessarily mean that oxytocin makes prosocials greedy, as we found that prosocials still have greater prosociality than individualists under oxytocin. This is also not likely to be a ceiling effect, as there is no oxytocin effect on increasing prosociality even when prosocials employ a more "self-centered" reference-point in a competitive context.

We found that oxytocin significantly reduces the strength of amygdala social value distance representations in prosocials and this was associated with a trend towards reduced prosociality. More sensitive changes in neural responses have often been observed in previous studies of prosocial behavior ${ }^{43}$. In the current study, one possible account is that social desirability or social pressure prevents prosocials from engaging in more self-centered performance. Although oxytocin significantly reduces amygdala representations of social values, consideration of both reputation ${ }^{44}$ and others' approval $^{45}$ may prevent neural effects from translating into explicit changes in behavior. Finally, in the within-subjects oxytocin replication experiment, we showed that the oxytocin effect on shifting the social reference-point towards greater prosociality varied as a function of an individual's disposition of social value orientation, suggesting that the dichotomous comparison in the between-subjects design may prevent identification of an effect that depends on individual disposition.

Oxytocin has been implicated in many social behaviors, from promoting trust, generosity and cooperation ${ }^{21,22,46,47}$ to aggravating mistrust and aggressive behavior ${ }^{48,49}$.

480 The variable nature of oxytocin effects on prosociality is increasingly recognized.

481 Seemingly contradictory oxytocin effects may be moderated by poorly understood 482 individual and contextual differences ${ }^{21,24}$. Our finding of distinct oxytocin effects on 483 the social reference-point for prosocials and individualists provides evidence for the 484 underlying computational and neural basis for oxytocin's effect on prosociality and 485 helps reconcile conflicting results in the literature. A concern in previous studies is that 486 post hoc explorations of different modulations by individual differences risk inflating 487 the rate of Type I errors ${ }^{50}$. The current study examined only a single a priori specified 488 modulator as we screened participants for social disposition before the oxytocin 
489

490

491

492

493

494

495

496

497

498

499

500

501

502

503

504

505

506

507

508

509

510

511

512

513

516

517

518

514 Neuroscience and Learning, IDG/McGovern Institute for Brain Research, Beijing

515 Normal University (to YM), and by the Max Planck Society and a MRC Career

experiment, which allowed us to specifically test for different effects of oxytocin in prosocials and individualists. We consistently found across multiple studies that the selective effect of oxytocin on promoting prosociality in individualists was present in both competitive and non-competitive contexts, in both within- and between-subjects designs and with different experimental task designs.

Taken together, our results reveal a neural mechanism that underlies social value representations, providing new insight into the processes that influence human social decisions. Our results demonstrate that oxytocin adaptively modulates social value representations in the amygdala and imply a fundamental role of oxytocin in social decision-making. These insights and the identification of a selective effect of oxytocin on prosociality in individualists may have implications for treating neuropsychiatric disorders with social deficits, including autism and sociopathy.

\section{METHODS}

Methods, including additional references, Nature Research reporting summaries, statements of data availability and any associated accession codes are available in the online version of the paper.

Acknowledgments. We thank H. Zhang, D. Wang, X. Zou and H. Wang for their assistance in data collection. We also thank M. Crockett and three anonymous reviewers for their constructive suggestions on an earlier draft of this manuscript. This work was supported by the National Natural Science Foundation of China (Projects $31722026 ; 91632118 ; 31771204 ; 31661143039$, to YM), the Fundamental Research Funds for the Central Universities (2017XTCX04; 2018EYT04, to YM), Open Research Fund of the State Key Laboratory of Cognitive Neuroscience, Beijing Normal University (to YM), startup funding from the State Key Laboratory of Cognitive

16 Development Award (MR/N02401X/1) to RBR.

17 Author Contributions. Y.M. conceived and designed the project, designed the fMRI experiment; Y.M. and Y.L. designed the replication experiments; W.Li., X.W., X.P. 
519 and Y.M. performed the fMRI experiment; S.L. and X.Y. performed the replication

520 experiments; Y.L., S.L., W.Lin. and Y.M. analysed the data and interpreted the results

521 of the fMRI and behavioral experiments; and Y.L., W.Lin., R.B.R. and Y.M. wrote the

522 paper.

523

524 Competing Interests Statement. The authors declare no competing interests.

525

526 


\section{References}

528 1. Sanfey, A.G. Social decision-making: insights from game theory and

529 neuroscience. Science 318, 598-602 (2007).

530 2. Rangel, A., Camerer, C. \& Montague, P.R. A framework for studying the

531 neurobiology of value-based decision making. Nat. Rev. Neurosci. 9, 545-556 (2008).

532 3. Haruno, M. \& Frith, C.D. Activity in the amygdala elicited by unfair divisions

533 predicts social value orientation. Nat. Neurosci. 13, 160-161 (2010).

534 4. Declerck, C.H., Boone, C. \& Kiyonari, T. The effect of oxytocin on cooperation

535 in a prisoner's dilemma depends on the social context and a person's social value

536 orientation. Soc. Cogn. Affect. Neurosci. 9, 802-809 (2014).

537 5. Van Lange, P.A. The pursuit of joint outcomes and equality in outcomes: An

538 integrative model of social value orientation. J. Pers. Soc. Psychol. 77, 337 (1999).

539 6. Chang, S.W., et al. Neuroethology of primate social behavior. Proc. Natl. Acad.

540 Sci. USA 110, 10387-10394 (2013).

541 7. Chang, S.W., et al. Neural mechanisms of social decision-making in the primate

542 amygdala. Proc. Natl. Acad. Sci. USA 112, 16012-16017 (2015).

543 8. Will, G.-J., Rutledge, R.B., Moutoussis, M. \& Dolan, R.J. Neural and

544 computational processes underlying dynamic changes in self-esteem. eLife 6 (2017).

545 9. Gottfried, J.A., O'Doherty, J. \& Dolan, R.J. Encoding predictive reward value in

546 human amygdala and orbitofrontal cortex. Science 301, 1104-1107 (2003).

547 10. Schultz, W., Dayan, P. \& Montague, P.R. A neural substrate of prediction and

548 reward. Science 275, 1593-1599 (1997).

549 11. Boccia, M.L., Petrusz, P., Suzuki, K., Marson, L. \& Pedersen, C.A.

550 Immunohistochemical localization of oxytocin receptors in human brain.

551 Neuroscience 253, 155-164 (2013).

552 12. Haber, S. N., \& Knutson, B. The reward circuit: linking primate anatomy and

553 human imaging. Neuropsychopharmacol, 35, 4 (2010).

554 13. Bickart, K. C., Dickerson, B. C., \& Barrett, L. F. The amygdala as a hub in brain

555 networks that support social life. Neuropsychologia 63, 235-248 (2014).

556 14. Paton, J.J., Belova, M.A., Morrison, S.E. \& Salzman, C.D. The primate amygdala

557 represents the positive and negative value of visual stimuli during learning. Nature

558 439, 865-870(2006). 
560 monetary loss aversion. Proc. Natl. Acad. Sci. USA 107, 3788-3792 (2010).

561 16. Garrett, N., Lazzaro, S.C., Ariely, D. \& Sharot, T. The brain adapts to dishonesty.

562 Nat. Neurosci. 19, 1727-1732 (2016).

563 17. Knoch, D., \& Fehr, E. Resisting the power of temptations. Ann. N. Y. Acad.

564 Sci. 1104, 123-134 (2007).

565 18. Spitzer, M., Fischbacher, U., Herrnberger, B., Grön, G. \& Fehr, E. The neural

566 signature of social norm compliance. Neuron 56, 185-196 (2007).

567 19. Rudebeck, P. H., Saunders, R. C., Prescott, A. T., Chau, L. S., \& Murray, E. A.

568 Prefrontal mechanisms of behavioral flexibility, emotion regulation and value

569 updating. Nat. Neurosci. 16, 1140 (2013).

570 20. Haruno, M., Kimura, M., \& Frith, C. D. Activity in the nucleus accumbens and

571 amygdala underlies individual differences in prosocial and individualistic economic

572 choices. J. Cogn. Neurosci. 26, 1861-1870 (2014).

573 21. Ma, Y., Shamay-Tsoory, S., Han, S. \& Zink, C.F. Oxytocin and social adaptation:

574 Insights from neuroimaging studies of healthy and clinical populations. Trends. Cogn.

575 Sci. 20, 133-145 (2016).

576 22. Zink, C.F. \& Meyer-Lindenberg, A. Human neuroimaging of oxytocin and

577 vasopressin in social cognition. Horm. Behav. 61, 400-409 (2012).

578 23. Alvares, G.A., Hickie, I.B. \& Guastella, A.J. Acute effects of intranasal oxytocin

579 on subjective and behavioral responses to social rejection. Exp. Clin.

580 Psychopharmacol. 18, 316 (2010).

581 24. Ma, Y., Liu, Y., Rand, D.G., Heatherton, T.F. \& Han, S. Opposing oxytocin

582 effects on intergroup cooperative behavior in intuitive and reflective minds.

583 Neuropsychopharmacol 40, 2379-2387 (2015).

584 25. Scheele, D., et al. Oxytocin modulates social distance between males and females.

585 J. Neurosci. 32, 16074-16079 (2012).

586 26. Chang, S.W., Barter, J.W., Ebitz, R.B., Watson, K.K. \& Platt, M.L. Inhaled

587 oxytocin amplifies both vicarious reinforcement and self reinforcement in rhesus

588 macaques (Macaca mulatta). Proc. Natl. Acad. Sci. USA 109, 959-964 (2012).

589 27. Lambert, B., Declerck, C.H., Boone, C. \& Parizel, P.M. A functional MRI study

590 on how oxytocin affects decision making in social dilemmas: Cooperate as long as it 
591 pays off, aggress only when you think you can win. Horm. Behav. 94, 145-152

592 (2017).

593 28. Murphy, R.O., Ackermann, K.A. \& Handgraaf, M. Measuring social value

594 orientation. Judgm. Decis. Mak. 6, 771-781 (2011).

595 29. Constantinescu, A.O., O’Reilly, J.X. \& Behrens, T.E. Organizing conceptual

596 knowledge in humans with a gridlike code. Science 352, 1464-1468 (2016).

597 30. Doeller, C.F., Barry, C. \& Burgess, N. Evidence for grid cells in a human

598 memory network. Nature 463, 657-661 (2010).

599 31. Evans, A.M. \& Rand, D.G. Cooperation and decision time. Curr. Opin. Psychol.

600 26, 67-71 (2018).

601 32. Krajbich, I., Bartling, B., Hare, T. \& Fehr, E. Rethinking fast and slow based on a

602 critique of reaction-time reverse inference. Nat. Commun. 6, 7455 (2015).

603 33. Neubert, F.X., Mars, R.B., Thomas, A.G., Sallet, J. \& Rushworth, M.F.

604 Comparison of human ventral frontal cortex areas for cognitive control and language

605 with areas in monkey frontal cortex. Neuron 81, 700-713 (2014).

606 34. Lim, S. L., O'Doherty, J. P., \& Rangel, A. The decision value computations in the

607 vmPFC and striatum use a relative value code that is guided by visual attention. $J$.

608 Neurosci. 31, 13214-13223 (2011).

609 35. Jocham, G., Hunt, L. T., Near, J., \& Behrens, T. E. A mechanism for value-guided

610 choice based on the excitation-inhibition balance in prefrontal cortex. Nat.

611 Neurosci. 15, 960 (2012).

612 36. Morishima, Y., Schunk, D., Bruhin, A., Ruff, C.C. \& Fehr, E. Linking brain

613 structure and activation in temporoparietal junction to explain the neurobiology of

614 human altruism. Neuron 75, 73-79 (2012).

615 37. Hutcherson, C.A., Bushong, B. \& Rangel, A. A Neurocomputational Model of

616 Altruistic Choice and Its Implications. Neuron 87, 451-462 (2015).

617 38. Strombach, T., et al. Social discounting involves modulation of neural value

618 signals by temporoparietal junction. Proc. Natl. Acad. Sci. USA 112, 1619-1624

619 (2015).

620 39. Kringelbach, M. L. The human orbitofrontal cortex: linking reward to hedonic

621 experience. Nat. Rev. Neurosci. 6, 691 (2005).

622 40. Ruff, C.C. \& Fehr, E. The neurobiology of rewards and values in social decision

623 making. Nat. Rev. Neurosci. 15, 549 (2014). 
624 41. Telzer, E. H., Fuligni, A. J., Lieberman, M. D., \& Galvan, A. Neural sensitivity to

625 eudaimonic and hedonic rewards differentially predict adolescent depressive

626 symptoms over time. Proc. Natl. Acad. Sci. USA 111, 6600-6605 (2014).

627 42. Mosher, C. P., Zimmerman, P. E., \& Gothard, K. M. Neurons in the monkey

628 amygdala detect eye contact during naturalistic social interactions. Curr. Biol. 24,

629 2459-2464 (2014).

630 43. Hein, G., Morishima, Y., Leiberg, S., Sul, S. \& Fehr, E. The brain’s functional

631 network architecture reveals human motives. Science 351, 1074-1078 (2016).

632 44. Ariely, D., Bracha, A., \& Meier, S. Doing good or doing well? Image motivation

633 and monetary incentives in behaving prosocially. Am. Econ. Rev. 99, 544-55 (2009).

634 45. Bénabou, R., \& Tirole, J. Incentives and prosocial behavior. Am. Econ. Rev. 96,

635 1652-1678 (2006).

636 46. Aydogan, G., et al. Oxytocin promotes altruistic punishment. Soc. Cogn. Affect.

637 Neurosci. 12, 1740-1747 (2017).

638 47. Yan, X., Yong, X., Huang, W., \& Ma, Y. Placebo treatment facilitates social trust

639 and approach behavior. Proc. Natl. Acad. Sci. USA 115, 5732-5737 (2018).

640 48. Ne'eman, R., Perach-Barzilay, N., Fischer-Shofty, M., Atias, A. \&

641 Shamay-Tsoory, S.G. Intranasal administration of oxytocin increases human

642 aggressive behavior. Horm. Behav. 80, 125-131 (2016).

64349 Radke, S. \& De Bruijn, E. The other side of the coin: oxytocin decreases the

644 adherence to fairness norms. Front. Hum. Neurosci. 6, 193 (2012).

645 50. Nave, G., Camerer, C., \& McCullough, M. Does oxytocin increase trust in

646 humans? A critical review of research. Perspect Psychol Sci 10, 772-789 (2015).

647

$648 \quad$ Figure legends.

649 Figure 1. Experimental design. (a) Participants were presented with monetary outcome 650 pairs specifying potential amounts of money (“+” indicated gain and “_” indicated loss)

651 received by themselves (labeled as self) and another player (labeled as other).

652 Participants had $3 \mathrm{~s}$ to rate their preferences from 1 (least preferable) to 4 (most

653 preferable) for each monetary outcome pair, followed by a 1-5 s jittered inter-trial

654 interval. Monetary outcomes for the self and other define an angle $\theta$, which samples the

655 space from $-90^{\circ}$ to $180^{\circ}$. (b) Mean standardized preference ratings across all 
656 participants were plotted against monetary allocations for the self and other. (c) Based on the preference ratings, we computed the "reference-point" in the social value

658

659

660

661

662

663

664

665

666

667

668

669

670

671

672

673

674

675

676

677

678

679

680

681

682

683

684

685

686

687

688

representation $\varphi$, closely related to the preferred allocation that best accounts for the participant's social preferences over all allocations. Higher values of $\varphi$ correspond to a stronger preference for allocations that benefit the partner relative to oneself, indicating greater prosociality.

Figure 2. Oxytocin boosts prosociality selectively in individualists. More prosocial reference-points for social value representations in prosocials than individualists in the fMRI during-scan experiment (a, 31 prosocials and 30 individualists, $\mathrm{p}=2.91 \times 10^{-7}$, under placebo), in the online-replication experiment (b, $n=315,160$ prosocials and 155 individualists, $\left.p=2.71 \times 10^{-19}\right)$, in the fMRI post-scan experiment (d, in a competitive context via framing the payoff in a "winner takes all" manner, $p=7.69 \times 10^{-4}$, under placebo), and in the oxytocin-replication experiment (e, 40 prosocials and 40 individualists, $p=9.78 \times 10^{-7}$, under placebo). In the online-replication experiment, the estimated social reference-point was positively correlated with individual SVO scores: the more prosocial the disposition, the higher the $\varphi(\mathbf{c}, n=315$, Pearson's $r=0.55, p=$ $\left.4.38 \times 10^{-26}\right)$. Moreover, intranasal oxytocin increased prosociality in individualists but not in prosocials, by moving their reference-point $\varphi$ towards a preference for more prosocial allocations in the fMRI during-scan experiment (a, $n=125$, individualists under placebo: $n=30$ males, under oxytocin: $n=29$ males; prosocials under placebo: $n$ $=31$ males, under oxytocin: $n=35$ males, $p=0.013$ ) and in the oxytocin-replication experiment (e, 40 prosocials and 40 individualists, $p=0.011$ ). Moreover, in the oxytocin-replication experiment, SVO scores were negatively correlated with the effect of oxytocin on social reference-point (f, $n=80$, Pearson's $r=-0.23, p=0.041$ ). The individual specific reference-point $\varphi<0^{\circ}$ indicates a preference for pairs with self-gain and other-loss; $\varphi=0^{\circ}$ indicates a preference for self-gain without consideration of other's outcome; $0^{\circ}<\varphi<90^{\circ}$ indicates a preference for self-gain/other-gain pairs; $\varphi>$ $90^{\circ}$ indicates a preference for self-loss/other-gain pairs). Error bars represented standard error of the mean across participants within each group $\left({ }^{*} p<0.05,{ }^{*} p<0.01\right.$, and $* * * p<0.001 ;$ n.s, not significant). 
690 Figure 3. Amygdala activity in prosocials ( $n=30$ males $)$ and right 1OFC activity in individualists ( $n=30$ males) encode social value distance relative to an

692 individual-specific reference-point. (a) Coronal view of activations in the amygdala 693 that encode the social value distance, the difference between potential and preferred 694 social allocations, was significantly stronger in prosocials than individualists under 695 placebo $(\mathrm{P}<0.05$, FWE-corrected at the cluster level after voxel-wise thresholding at $\mathrm{P}$ $696<0.001$ ). (b) Axial view of right 1OFC activity encoding social value distance to a 697 greater degree in individualists than prosocials (voxel-wise threshold $\mathrm{p}<0.001$, small 698 volume correction $\mathrm{p}<0.05$ for an anatomically defined right 1OFC mask). Independent-samples $t$ test was used in (a) and (b) on the beta estimates from ROIs of amygdala $(\mathrm{t}(58)=2.75, \mathrm{p}=0.008,95 \% \mathrm{CI}=[0.05,0.33])$ and right $1 \mathrm{OFC}(\mathrm{t}(58)=$ $-2.46, \mathrm{p}=0.017,95 \% \mathrm{CI}=[-0.04,-0.004])$. The amygdala and right 1OFC ROIs employed anatomically defined masks (amygdala based on AAL bilateral anatomical mask, an anatomically defined right 1OFC mask, using combined connectivity-based parcellations 8-11 covering right $1 \mathrm{OFC}^{33}$ ).

Figure 4. Oxytocin promotes amygdala activity in representing social values in individualists $\left(n_{\text {placebo }}=30, n_{\text {oxytocin }}=26\right.$ in individualists, $n_{\text {placebo }}=30, n_{\text {oxytocin }}=30$ in prosocials). (a) Coronal view of amygdala showing interaction effect between Treatment and Social Disposition (peak voxels in right amygdala, FWE-corrected

$711 p=0.02$ ). Social Disposition-by-Treatment ANOVA showed significant interaction on 712 the beta estimates from anatomically defined amygdala $(F(1,112)=12.536, p=$

$7135.83 \times 10^{-4}, \eta^{2}=0.057$ ). (b) Trial-by-trial amygdala responses in predicting preference 714 rating, after controlling for predicted social value distance for each trial. The Social 715 Disposition-by-Treatment ANOVA on the trial-by-trial correlation coefficient showed 716 a significant interaction between Social Disposition and Treatment $(F(1,115)=6.722$, $\left.717 p=0.011, \eta^{2}=0.057\right)$. Error bars represented standard error of the mean across 718 participants within each group, ${ }^{*} p<0.05$ and ${ }^{*} p<0.01$. 


\section{Participants}

722 For the oxytocin-fMRI and behavioral oxytocin-replication experiments, we recruited

723 only male participants to avoid potential confounds of sex differences in oxytocin

724 effects $^{21,51}$, consistent with previous studies examining oxytocin effects on social

725 cognition. All participants had normal or corrected-to-normal vision and reported no

726 history of neurological or psychiatric diagnoses, or medication, drug or alcohol abuse.

727 Participants provided informed consent after the experimental procedure had been fully

728 explained and were informed of their right to withdraw at any time during the study.

729 The experimental protocol was in line with the standards of the Declaration of Helsinki

730 and approved by the research ethics committee at the State Key Laboratory of

731 Cognitive Neuroscience and Learning, Beijing Normal University (Beijing, China).

732 Oxytocin fMRI experiment. There were 282 male college students (mean age $=22.3 \pm$

7332.12 years) that participated in this study as paid volunteers. Participant's disposition in

734 social value orientation was measured in the behavioral session (149 prosocials and 83

735 individualists were identified). Among these, 127 participants were qualified and

736 willing to participate in the fMRI experiment (at least 7 days after the behavioral

737 session). Two participants (1.6\%) were excluded due to technical issues during

738 scanning, leaving 125 participants in the behavioral analysis (individualists under

739 placebo: $n=30$ males, mean age $22.2 \pm 2.35$ years, under oxytocin: $n=29$ males, mean

740 age $21.7 \pm 2.39$ years; prosocials under placebo: $n=31$ males, mean age $22.1 \pm 2.70$

741 years, under oxytocin: $n=35$ males, mean age $22.1 \pm 2.70$ years). An additional 9

742 participants (7.2\%) were excluded from further fMRI analysis due to excessive head

743 movement during scanning $(>3 \mathrm{~mm})$, leaving 116 participants for fMRI data analysis.

744 In the end, there were 60 prosocials including 30 administered placebo (mean age, 22.7

$745 \pm 2.61$ years) and 30 administered oxytocin (mean age, $21.8 \pm 2.38$ years), and 56

746 individualists including 30 administered placebo (mean age, $23.0 \pm 2.29$ years) and 26

747 administered oxytocin (mean age, $22.5 \pm 3.03$ years) in the formal fMRI data analysis.

748 Prosocials and individualists receiving oxytocin or placebo were matched on state and

749 trait anxiety, depression, subjective well-being and happiness ratings (all $p>0.05$ on

750 both the main and interaction effects of Treatment and Social Disposition;

751 Supplementary Table 3). 
752 The sample size of the fMRI study was determined prior to data collection. We 753 conducted sample size estimation using $\mathrm{G}^{*}$ Power $3.1^{52}$ to determine the number of 754 participants sufficient to detect a reliable effect. Based on an estimated average 755 small-to-medium effect size of oxytocin effect on social behaviors (Cohen's $d=0.28$ )

75653.104 participants were needed to detect a significant effect $(\alpha=0.05, \beta=0.80$,

757 two-by-two mixed ANOVA interaction effects). We planned to recruit 125 participants 758 (assuming 10-20\% participants would be removed from the fMRI data analysis due to 759 excessive head movement). In the end, we recruited 127 participants because the 41th 760 and 42th participants did not complete the experiment due to technical issues during 761 scanning. For comparison, we also considered the 58 oxytocin-fMRI studies published 762 at the time we initiated our experiment in June 2015, of which 23 employed 763 between-subject design recruiting healthy individuals. On average, the sample size was 76450.89 in total, 25.82 for the placebo group and 25.47 for the oxytocin group. Thus, our 765 planned sample size of 125 participants was a decent sample size compared to the 766 average across oxytocin-fMRI studies. Moreover, the sample size of 116 participants 767 (after removal of subjects due to technical issues and excessive head movement) was 768 adequate to reveal reliable effects, exceeding the 104 participants needed for $80 \%$ 769 power. Oxytocin-replication experiment. We conducted an additional behavioral experiment 771 for the replication of the oxytocin effect using a double-blind, randomized, 772 placebo-controlled, within-subjects crossover design. The sample size was 773 predetermined based on the effect size (Cohen's $d=0.45)$ from our original finding in 774 the fMRI study. The $G^{*}$ Power calculation suggested that 40 participants (20 for each 775 group) were required to detect a reliable effect $(\alpha=0.05, \beta=0.80$ for a within (oxytocin 776 vs. placebo)-between (prosocial vs. individualist) interaction). To obtain a better sense 777 of the robustness of the original findings, we doubled the estimated sample size, aiming 778 to enroll 40 participants per group, with corresponding power equal to $98 \%$. We 779 replicated the selective oxytocin effects on promoting prosociality (i.e., $\varphi$ ) in 780 individualists in the whole sample (40 prosocials and 40 individualists), as well as in 781 the first 20 prosocials and 20 individualists (as estimated by the $\mathrm{G}^{*}$ Power analysis). 782140 males (mean age, $22.33 \pm 3.35$ years) were invited to a behavioral session to 783 identify their disposition in social value orientation. Among these, 82 participants were 
784 qualified and willing to participate in the oxytocin experiment (at least 7 days after the 785 first behavioral session). Two participants did not show up for the second session. Thus, 78680 participants (40 prosocials, mean age, $22.08 \pm 3.47$ years; 40 individualists; mean 787 age, $21.54 \pm 2.42$ years) were included in the final data analysis.

Online-replication experiment. We conducted an online experiment with a large sample $(n=315,132$ males, 160 prosocials, mean age $=22.40 \pm 3.27 ; 155$ individualists, mean age $=22.48 \pm 3.30$ ) to provide a replication for our finding that the social reference model outperforms other models. Prosocials and individualists did not differ in their ratings on the first impression, likeability and attractiveness of the online partner (independent-samples $t$ test, impression: $t_{313}=1.03, p=0.305$; likeability: $t_{313}$ $=0.98, \mathrm{p}=0.328$; attractiveness: $\mathrm{t}_{313}=0.73, \mathrm{p}=0.465$.

Procedure.

798 Participants were first invited to the behavioral session to identify their social

799 disposition and be screened for eligibility of the fMRI and oxytocin behavioral

800 experiments. Participants recruited in the fMRI experiment were randomly assigned to

801 the intranasal administration of oxytocin or placebo in a double-blind

802 placebo-controlled between-subjects design. In the oxytocin experiment, participants

803 received either oxytocin or placebo intranasally in two separate sessions, with a

804 5-7-day washout period between two sessions. The order of oxytocin and placebo

805 treatment was counterbalanced across participants. All participants were instructed to

806 abstain from cigarette, alcohol and caffeine during the 24 hours prior to the experiment,

807 and to refrain from eating or drinking anything except water for 2 hours before the

808 experiment. Participants self-administrated oxytocin or placebo $35 \mathrm{~min}^{54}$ before the

809 main task, i.e., a monetary outcome-pair evaluation task (a revised one with monetary

810 pairs sampled on 3 circles of different circumference was used in the

811 oxytocin-replication experiment).

812 Social disposition measurements. In the fMRI and the oxytocin-replication

813 experiments, participants were first invited to a behavioral session to identify their 
814 dispositions in social preference. In the behavioral session, all participants provided

815 demographic information and completed the triple dominance (TD) ${ }^{5}$ and social value

816 orientation $(\mathrm{SVO})^{28}$ tasks, which were conventional measurements of one's stable

817 disposition in social value orientation. To incentivize authentic responses during social

818 interactions, participants were recruited in groups of 8-10 individuals (all were

819 strangers to each other). For each economic game, participants were paired with a new,

820 mutually anonymous partner.

821 The TD task is a 9-item measure of one's social disposition by asking participants to

822 choose from 3 types of hypothetical self-other monetary allocation options (e.g.,

823 prosocial option: self $=100$, other $=100$; individualistic option: self $=110$, other $=60$;

824 and competitive option: self $=100$, other $=20$ ). Based on their decisions to the 9 items,

825 participants were classified as prosocial (who chose prosocial options on 6 or more

826 items), individualist (who chose individualistic options on 6 or more items), or

827 competitor (who chose competitive responses on 6 or more items). Participants who

828 failed to choose the same type of options on at least 6 items were referred to as

829 "unidentified". In the current study, we referred to both "individualist" and "competitor"

830 as "individualists" in comparison to "prosocial".

831 The SVO slider measure included 6 primary items and 9 secondary items. For each

832 item, participants were asked to choose the most preferred one from 9 monetary

833 allocation choices over a well-defined continuum of joint payoffs. Based on the inverse

834 tangent of the ratio between mean allocations for the self and the paired partner, the 6

835 primary items yielded a measure that categorized participants into: altruist, prosocial,

836 individualist, and competitor ${ }^{28}$. Here, we referred to both "altruist" and "prosocial" as

837 "prosocials" (i.e., $\mathrm{SVO}^{\circ}>22.45^{\circ}$ ), and both "individualist" and "competitor" as

838 "individualists" (i.e., $\mathrm{SVO}^{\circ}<22.45^{\circ}$ ). The scores of 9 secondary items of SVO are used

839 to calculate an independent measure of inequality aversion, i.e., the general preference

840 for fairness and resistance to inequalities. To ensure a reliable measure of social

841 disposition, only participants who were consistently classified by the TD and SVO

842 tasks were deemed "qualified" (either "prosocial" or "individualist").

843 Prosocial behavior measures. Participants were also invited to the public goods game

844 (PGG) and the dictator game (DG). The contribution participants made in these two 
845 games have been separately used as indicators of the levels of cooperation and altruism

846 - two key characteristics of prosocial behaviors ${ }^{24,55-57}$.

847 In the 4-player PGG, participants initially receive 80 experimental monetary units (MU)

848 and decide the amount of MU to contribute to a 4-player common project vs. to keep for

849 themselves. The money contributed to the common project would be doubled and

850 evenly divided among the 4 players. The final payoff is equal to the sum of money they

851 keep for the self and money split from the common project. The amount of money

852 contributed to the common project reflects cooperative behavior.

853 In the DG, "the dictator" (i.e., the participant), determines how to split 80 MU between

854 himself and another player. The other players, "the recipient", simply receive the

855 remainder of the endowment left by the dictator. The recipient's role is entirely passive

856 and has no input into the outcome of the game. The amount the dictator sent to the

857 recipient indicates his altruistic behavior.

858

859 fMRI session. A pair of participants, who were strangers to each other, was invited to

860 the fMRI experiment at the same time. Upon arrival, participants' mood was measured

861 using Positive and Negative Affect Scale (PANAS), which was later measured again

862 after the experiment to quantify potential mood change. There was no significant mood

863 change overall and no significant interaction effect with division of Social Disposition

864 or Treatment (Supplementary Table 4). We measured participants' salivary oxytocin

865 baseline levels by collecting their salivary samples before oxytocin or placebo

866 administration (Supplementary Fig. 4). There was no significant main effect or

867 interaction effect between Social Disposition and Treatment on the salivary oxytocin

868 level. Each pair of participants was given $5 \mathrm{~min}$ to introduce themselves to each other to

869 strengthen the oxytocin effect on social cognition ${ }^{58}$. We ensured that participants

870 introduced their names to each other, which were also presented on the screen for each

871 monetary allocation. Participants in each pair were scanned in sequence and randomly

872 treated with oxytocin or placebo. The procedure of oxytocin or placebo administration

873 was similar to previous research ${ }^{24}$. A single dose of 24 IU oxytocin or placebo

874 (containing the active ingredients except for the neuropeptide) was intranasally

875 self-administered by nasal spray approximately $35 \mathrm{~min}$ before the fMRI scanning under

876 an experimenter's supervision. The spray was administered to participants three times 
877 with each administration consisted of one inhalation of 4 IU into each nostril. The

878 choice of 24 IU oxytocin and its effect on brain oxytocin level is explained in

879 Supplementary Note 1. After scanning, participants were asked to perform a similar

880 post-scan monetary outcome-pair evaluation task in a competitive context. The

881 duration of the fMRI scanning and the post-scan test were carefully controlled within

882 the time frame of the oxytocin peak response in the brain ${ }^{54}$.

883 Monetary outcome-pair evaluation task during MRI scanning. In the MRI scanner,

884 participants were presented with pairs of monetary outcomes assigned to the self and

885 the paired participant (referred as partner). Participants evaluated their preference of

886 each monetary allocation on a 4-point Likert scale ( $1=$ least preferable to $4=$ most

887 preferable) by a button press. To encourage genuine responses and minimize the

888 influences of social norms or social pressure, the preference ratings were unknown to

889 the other player. Participants were told that their preference rating for each monetary

890 outcome pair would determine the overall gains for self $\left(G_{s}\right)$ and the partner $\left(G_{p}\right)$,

891 i.e., $\mathrm{G}_{\mathrm{s}}=\sum \mathrm{ms}_{\mathrm{i}}{ }^{*} \mathrm{p}_{\mathrm{i}}$, and $\mathrm{G}_{\mathrm{p}}=\sum \mathrm{mp}_{\mathrm{i}}{ }^{*} \mathrm{p}_{\mathrm{i}}$, where $\mathrm{p}_{\mathrm{i}}$ is participants' preference rating for the

892 monetary outcome pair, $\mathrm{i} ; \mathrm{ms}_{\mathrm{i}} / \mathrm{mp}_{\mathrm{i}}$ is the monetary amount for self or the partner in

893 monetary pair, i. In each trial, the monetary allocation was presented for $3 \mathrm{~s}$, followed

894 by a jittered time interval, pseudo-randomized from $1 \mathrm{~s}$ to $5 \mathrm{~s}$ (with mean interval of $3 \mathrm{~s}$;

895 Fig. 1a). There were two sessions with 90 trials per session, presented in a random

896 order.

897 To determine appropriate monetary allocations for the fMRI scanning, we first

898 conducted a pilot behavioral experiment on an independent sample $(n=60)$, where we

899 included the full space of monetary outcome pairs and asked participants to rate their

900 preference for each allocation on a 9-point Likert scale $(1=$ least preferable; $9=$ most

901 preferable). We found that participants reported invariably with the least preferable for

902 pairs in the third quadrants and along the negative $\mathrm{X}$ - or $\mathrm{Y}$-axis, where both self and the

903 partner lose money (average preference rating of 1.8 on a 1-9 scale, with no rating

904 scores higher than 3). Therefore, these pairs (i.e., $\$$ Self $\leq 0$ and/or $\$$ Other $\leq 0$ ) were not

905 included in the fMRI task. The monetary outcome pairs for self and the partner, as

906 illustrated in Fig. 1a, were designed in a way as to form angles that evenly sampled

907 from $-90^{\circ}$ to $180^{\circ}$ with an interval approximately $5^{\circ}$, based on an egocentric

908 reference-point (i.e., $0^{\circ}$, which indicates perfect alignment with the positive $\mathrm{X}$-axis). 
909 We also included pairs where only the self or the partner gained money (evenly

910 sampled along the positive $\mathrm{X} / \mathrm{Y}$-axis) while the opponent received zero. These pairs

911 were used to generate functional masks for fMRI ROI analysis and were not included in

912 formal behavioral analyses.

913 Monetary outcome-pair evaluation task in a competitive context. Participants

914 completed a post-scan behavioral experiment largely the same as that in the fMRI task,

915 but with two key differences. First, participants reported their preference of each

916 monetary outcome pair on 10 instead of 4 levels $(0=$ least preferable to $9=$ most

917 preferable). Second, we induced a self-interest and other-interest conflict situation by

918 framing the payoff in a competitive context, where participants would get a bonus

919 reward if and only if the sum of gains to the themselves was larger than that to the

920 partner. Otherwise, they gained nothing (i.e., "winner takes all"). Therefore, the self

921 and partner's interest were in direct competition in this context. There was one session

922 with 90 trials presented in a random order.

923 Monetary outcome-pair evaluation task in oxytocin-replication and online replication

924 experiments. The task design for the replication experiments was identical to the fMRI

925 experiment except that the monetary pairs were sampled on 3 circles of different

926 circumference (radius $=5,6,9$ ), with $\theta$ ranging from $-90^{\circ}$ to $180^{\circ}$ with different

927 intervals $\left(5^{\circ}, 17^{\circ}, 23^{\circ}\right)$. There was one session with 82 trials presented in a random

928 order.

929 Behavioral analysis. We constructed 8 behavioral models based on theoretical

930 considerations (Supplementary Fig. 2). For the social reference model (the winning

931 model, Supplementary Fig. 2), we modeled z-scored preference ratings (P) for each

932 participant: $\mathrm{P}=\beta 1 * \cos (\theta)+\beta 2 * \sin (\theta)$. In this model, $\beta 1$ and $\beta 2$ are weights for how

933 much people care about the value of a potential payoff for themselves (\$Self) and for

934 the partner (\$Other), respectively. The angle $\theta$ depends on the difference between those

935 values. We then computed a single individual-specific reference-point $\varphi$ for each

936 participant based on the ratio of $\beta 1$ and $\beta 2: \varphi=\operatorname{atan}(\beta 2 / \beta 1)^{29,30}$ (Fig. 1c). The social

937 value distance reflects the difference between a potential self-other allocation $\theta$ and a

938 preferred allocation $\varphi$ that reflects an individual-specific reference-point against which

939 potential allocations are compared. 
940 When all monetary pairs lie on the circumference of a circle, $\cos (\theta)$ can be seen simply

941 as the amount offered to the self and $\sin (\theta)$ as the amount offered to the other, divided

942 by the radius of the circle. However, when including monetary pairs from circles with

943 different radii (i.e., the modified design used in additional experiments), $\cos (\theta)$ and

$944 \sin (\theta)$ provide a compact index that permits investigation of the relationship between

945 self and other in a value-insensitive way $\left(\right.$ since $\left.\cos (\theta)^{2}+\sin (\theta)^{2}=1\right)$.

946

947 fMRI acquisition and preprocessing.

948 Imaging acquisition. Whole-brain imaging data was collected on a GE 3-Tesla MR

949 scanner with a standard head coil (HDx, Signa MR 750 System; GE Healthcare,

950 Milwaukee, WI). Functional images were collected using an echo-planar imaging

951 sequence (axial slices, 32; slice thickness, 4 mm; gap, 1 mm; TR, 2000 ms; TE, 30 ms;

952 voxel size, $3.75 \times 3.75 \times 5 \mathrm{~mm}$; flip angle, $90^{\circ}$; FOV, $240 \times 240 \mathrm{~mm}$; and 285 volumes

953 for each session, two sessions in total). Structural images were acquired through 3D

954 sagittal T1-weighted magnetization-prepared rapid gradient echo (180 slices; TR,

$9558.208 \mathrm{~ms}$; TE, $3.22 \mathrm{~ms}$; slice thickness, $1 \mathrm{~mm}$; voxel size, $0.47 \times 0.47 \times 1.0 \mathrm{~mm}^{3}$; flip

956 angle, $12^{\circ}$; inversion time, $450 \mathrm{~ms}$; FOV , $240 \times 240 \mathrm{~mm}$ ).

957 Imaging preprocessing. Brain imaging data was preprocessed using Statistical

958 Parametric Mapping (SPM12; http://www.fil.ion.ucl.ac.uk/spm). The first 5 functional

959 images from each session were discarded for signal equilibrium and participants'

960 adaptation to scanning noise. Remaining images were corrected for slice acquisition

961 timing and realigned for head motion correction. Subsequently, functional images were

962 coregistered to each participant's grey matter image segmented from corresponding

963 high-resolution T1-weighted image, then spatially normalized into a common

964 stereotactic Montreal Neurological Institute (MNI) space and resampled into 2-mm

965 isotropic voxels. Finally, images were smoothed by an isotropic 3D Gaussian kernel

966 with 8-mm full-width at half-maximum.

967 GLM analysis. After preprocessing, we estimated parameters of different general linear

968 models (GLMs). All models included regressors for monetary outcome pair

969 presentation separately for trials on and off the $\mathrm{X} / \mathrm{Y}$ axis, button press, instructions, six

970 nuisance regressors for motion-related artefacts, and various parametric modulations 
971 associated with these regressors (detailed below). Parametric regressors were not

972 orthogonalized in the design matrix, ensuring that parameter estimates were not

973 confounded by spurious correlations due to signals related to other regressors ${ }^{59}$. All

974 regressors (parametrically modulated or not) were convolved with the canonical

975 hemodynamic response function (HRF) in SPM before entering the GLM. Data were

976 high-pass filtered at 1/128 Hz. We controlled for decision times for all fMRI analyses.

977 We created parametric regressors that were associated with the value distance or value

978 difference between self and other, at the monetary outcome-pair presentation to search

979 for brain regions that encoded the subjective distance in social value representation. In

980 the fMRI analysis, we included 10 GLM models: a social value distance from an

981 individual-specific reference-point (i.e., $1-\cos (\theta(t)-\varphi), \theta(t)$ is the angle of a potential

982 allocation at trial $\mathrm{t}$ and $\varphi$ is the individual-specific reference-point derived from our

983 social reference model), an egocentric reference (i.e., $\cos (\theta(\mathrm{t}))$, an allocentric

984 reference (i.e., $\sin (\theta(t))$, an objective equality reference-point (i.e., $\cos \left(\theta(t)-45^{\circ}\right)$,

985 monetary outcome for the self (i.e., \$Self), monetary outcome for the partner (i.e.,

986 \$Other), absolute value difference (|\$Self - \$Other) or advantageous (i.e., max (0, \$Self

987 -\$Other)) or disadvantageous inequality aversion (i.e., max (0, \$Other -\$Self))

988 separately, or with preference rating as parametric modulator in the GLM.

989 In building different GLMs, we are not arguing that the social reference model is

990 superior to other models in all environments nor claiming the dissimilarity measure is

991 the best measure for capturing amygdala responses as this was not our aim ${ }^{60}$. Rather,

992 we aimed to identify brain regions that could specifically represent deviations from the

993 reference-point of social preference.

994 Coefficients for each regressor were estimated for each participant using maximum

995 likelihood estimates to account for serial correlations in the data. Statistical

996 significance was determined at the group level using a random-effects analysis.

997 Significant clusters from second-level analyses were determined using a height

998 threshold of $P<0.001$ and an extent threshold of $P<0.05$ with cluster-based

999 family-wise error (FWE) correction. We also applied voxelwise inference using the

1000 FWE-corrected threshold of $P=0.05$ on the whole-brain analysis, given recent concern

1001 over cluster-wise inferences. For the relationship between value distance $(1-\cos (\theta(t)-$ 
$1002 \varphi$ ) and neural responses during monetary outcome-pair presentation, the peak voxels in

1003 right amygdala survived voxelwise FWE correction $(P=0.02)$.

1004 Control analysis of amygdala responses. One alternative hypothesis of the amygdala 1005 activity pattern is that it encoded \$Other or \$Disadvantageous Inequality, instead of the 1006 dissimilarity distance to the "reference-point" in our winning model, we reran the fMRI

1007 analysis from the first level controlling for \$Other and \$Disadvantageous Inequality as 1008 regressors in the GLM (without orthogonalization between regressors). We looked for 1009 the unique variance that can be explained by the dissimilarity distance to social 1010 reference-point over and beyond the variance explained by $\$$ Other or 1011 \$Disadvantageous Inequality. The main result of a significant Social Disposition $\mathrm{x}$

1012 Treatment interaction on the amygdala activity in coding deviations from the social 1013 reference-point was unchanged.

1014 We further tested another possibility of the amygdala response: it encoded \$Other in 1015 proportion to its importance to the individual. To test this possibility, we built another 1016 GLM model with the parametric regressor: $\beta 2 * \$$ Other, where $\beta 2$ represented the 1017 estimated weight of \$Other on social preference, reflecting the individual-specific 1018 importance of \$Other in social preference evaluation for each participant. However, the 1019 amygdala activity did not simply encode \$Other to the extent that it predicts social 1020 preferences, either at the whole brain level (height $p<0.001$, cluster-wise FWE, $p<$ 1021 0.05) or ROI (anatomically-defined) level.

1022 Statistics. The oxytocin-fMRI and oxytocin-replication experiments were 1023 double-blind, i.e., both participants and experimenters were blind to experimental 1024 conditions (both treatment and social disposition conditions). Data analysis was not 1025 performed blind to the conditions of the experiments. We first conducted one-way 1026 ANOVA with Social Disposition as a between-subjects factor to compare the social 1027 reference-point between individualists and prosocials under placebo. To evaluate the 1028 oxytocin effect on the social value representation, we conducted ANOVAs on 1029 behavioral and fMRI data, with Social Disposition (prosocial vs. individualistic) and 1030 Treatment (oxytocin vs. placebo) as between-subjects factors, followed by planned 1031 two-tailed $t$ tests to examine oxytocin effect separately in individualists and prosocials 
1032 (independent-samples $t$ test for fMRI study and paired-samples $t$ test for the

1033 oxytocin-replication study). Data distribution was assumed to be normal but this was

1034 not formally tested. All correlations were performed by Pearson's correlation

1035 coefficient analysis.

1036 Data Availability Statement. The data that support the findings of this study and the 1037 analysis code are available from the corresponding author upon reasonable request.

1038 Code availability. Analysis code to model the social value representation based on

1039 preference rating data is provided in the Supplementary Software.

1040

1041 Life Sciences Reporting Summary. Further information on research design is

1042 available in the Life Sciences Reporting Summary linked to this article.

1043 
51. Gao, S., et al. Oxytocin, the peptide that bonds the sexes also divides them. Proc. Natl. Acad. Sci. USA 113, 7650-7654. (2016).

1051 52. Faul, F., Erdfelder, E., Buchner, A. \& Lang, A.-G. Statistical power analyses

1052 using G*Power 3.1: Tests for correlation and regression analyses. Behav. Res.

1053 Methods. 41, 1149-1160 (2009).

1054 53. Walum, H., Waldman, I.D. \& Young, L.J. Statistical and Methodological

1055 Considerations for the Interpretation of Intranasal Oxytocin Studies. Biol. Psychiatry.

1056 79, 251-257 (2016).

1057 54. Paloyelis, Y., et al. A Spatiotemporal Profile of In Vivo Cerebral Blood Flow 1058 Changes Following Intranasal Oxytocin in Humans. Biol. Psychiatry. 79, 693-705 1059 (2016).

1060 55. Rand, D.G., Greene, J.D. \& Nowak, M.A. Spontaneous giving and calculated 1061 greed. Nature 489, 427-430 (2012).

1062 56. Fehr, E. \& Fischbacher, U. The nature of human altruism. Nature 425, 785-791 1063 (2003).

1064 57. Harbaugh, W.T., Mayr, U. \& Burghart, D.R. Neural responses to taxation and 1065 voluntary giving reveal motives for charitable donations. Science 316, 1622-1625 1066 (2007).

1067 58. Declerck, C.H., Boone, C. \& Kiyonari, T. Oxytocin and cooperation under 1068 conditions of uncertainty: the modulating role of incentives and social information.

1069 Horm. Behav. 57, 368-374 (2010).

1070 59. Andrade, A., Paradis, A.-L., Rouquette, S. \& Poline, J.-B. Ambiguous results in 1071 functional neuroimaging data analysis due to covariate correlation. Neuroimage 10, 1072 483-486 (1999). 
1073 60. Wilson, R.C. \& Niv, Y. Is model fitting necessary for model-based fMRI? PLoS.

1074 Comput. Biol. 11, e1004237 (2015).

1075

1076 

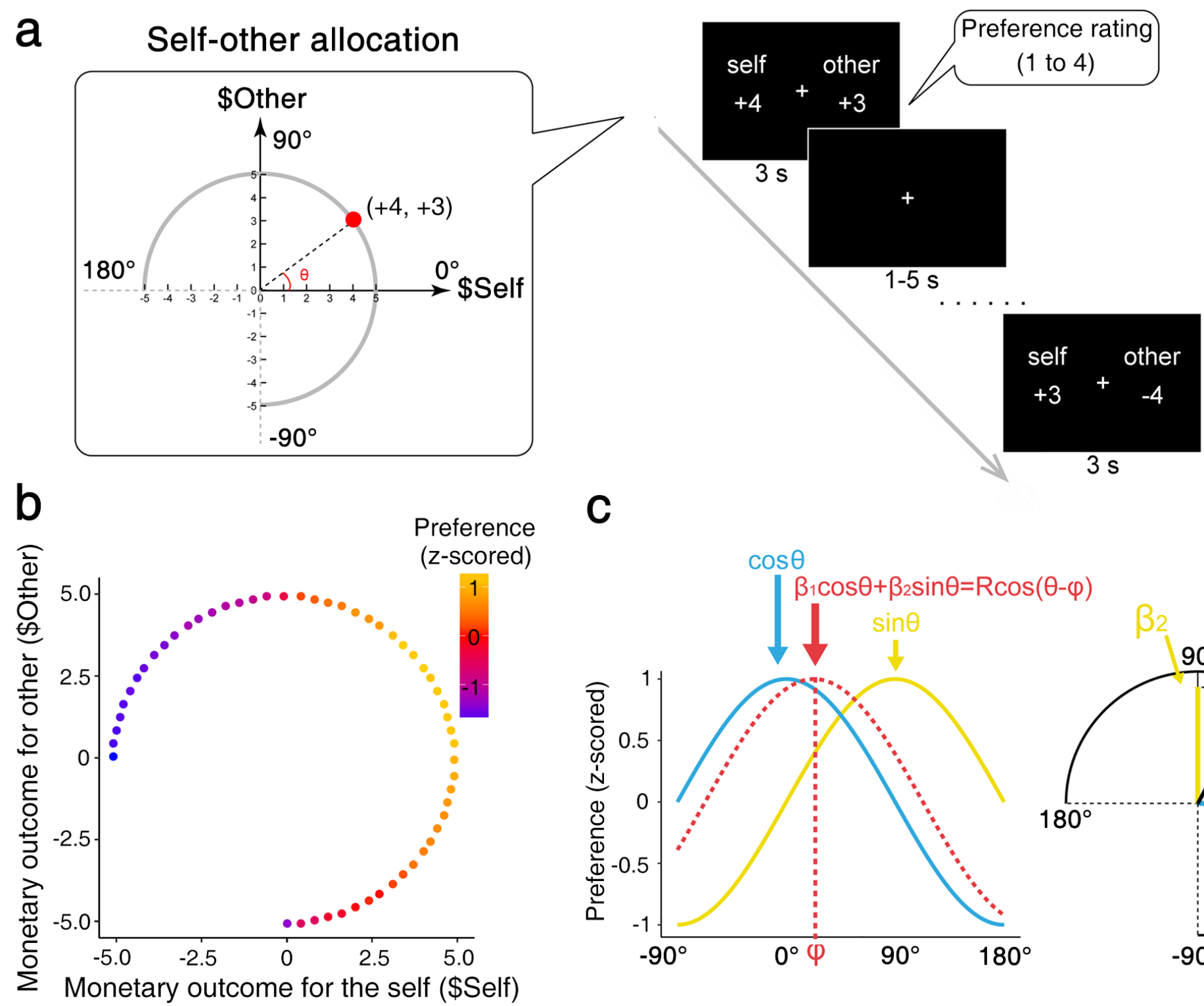

C

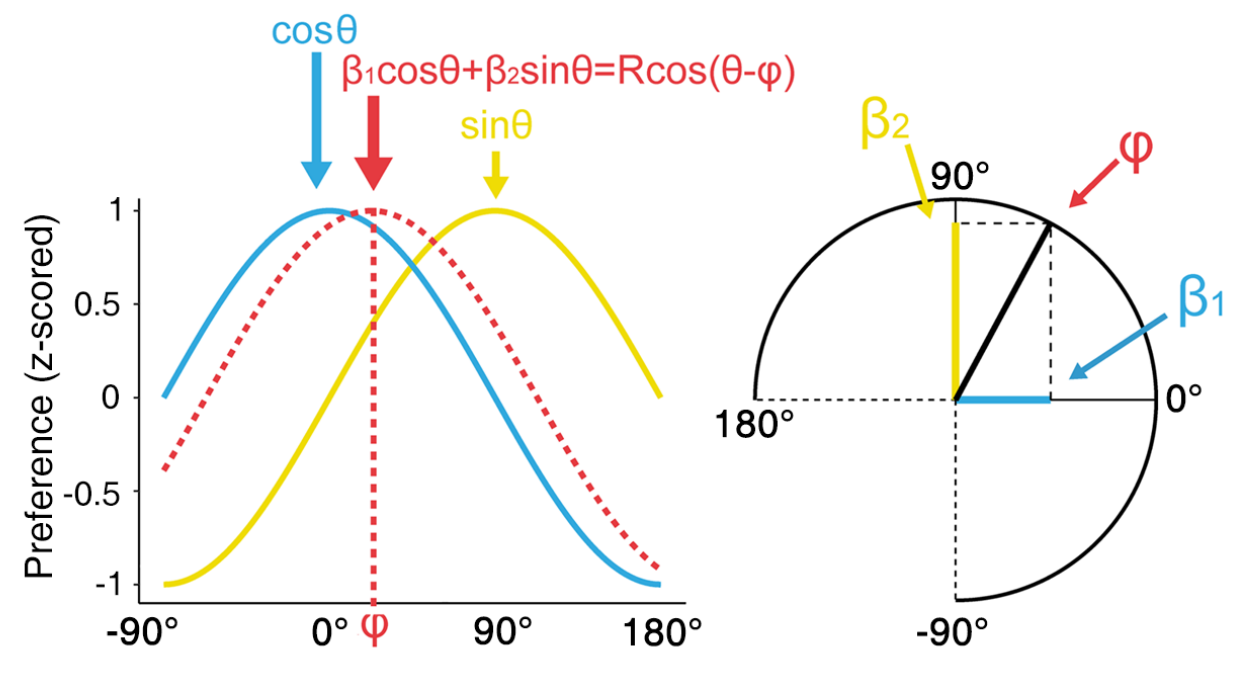


a fMRI during-scan

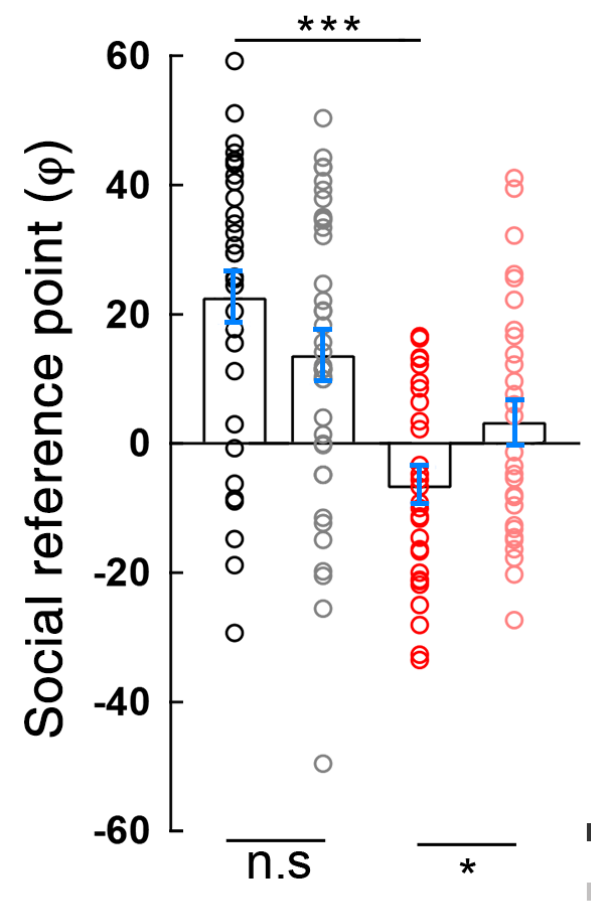

d fMRI post-scan

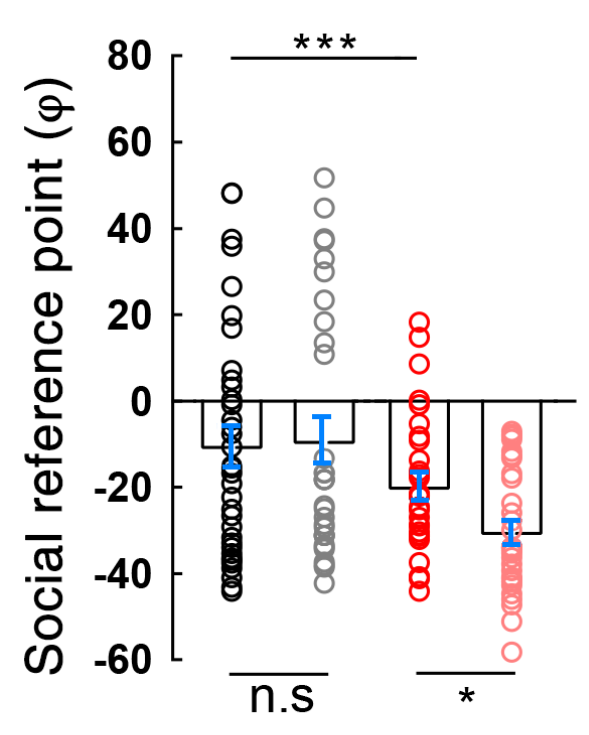

b Online-replication

C

Online-replication
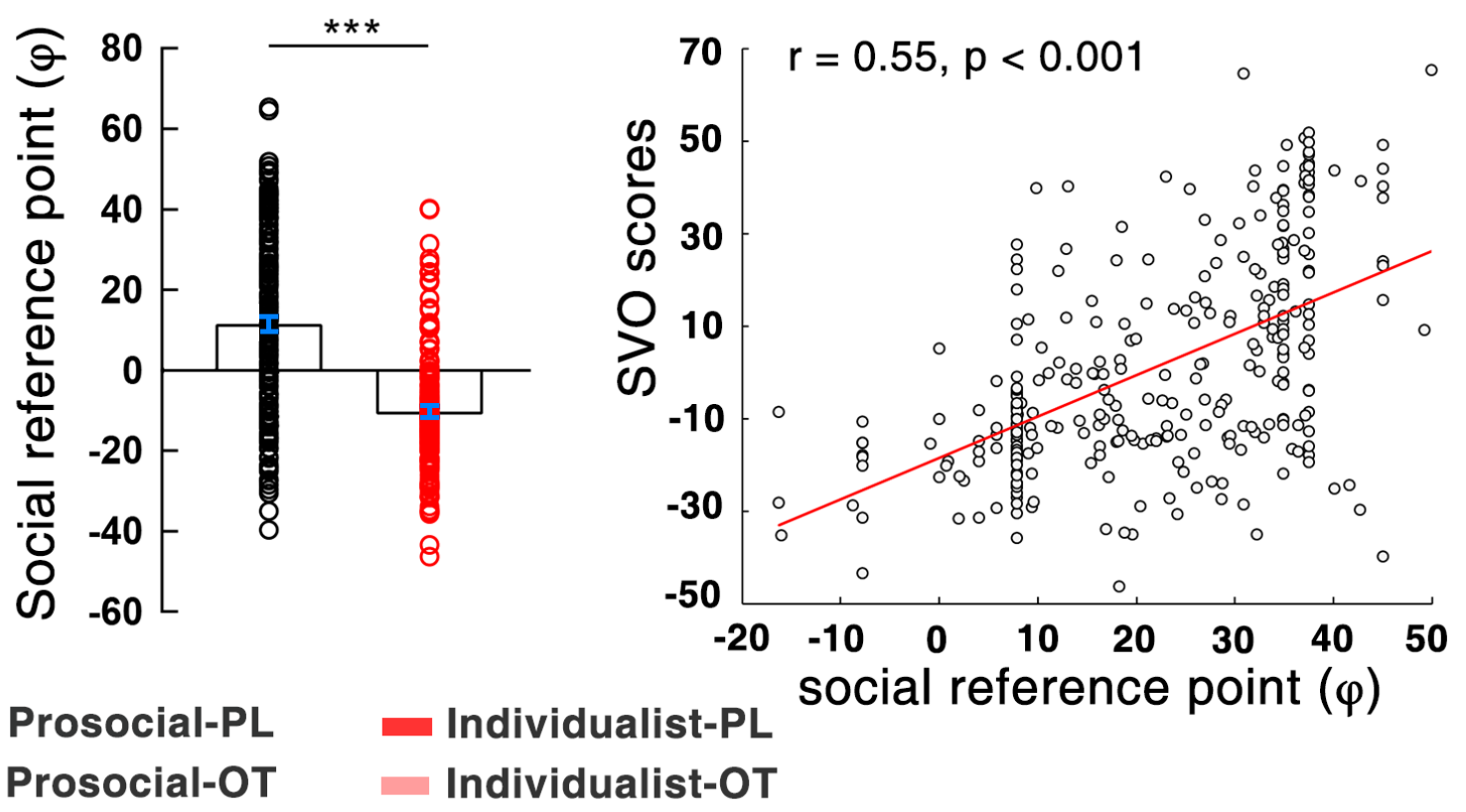

e Oxytocin-replication

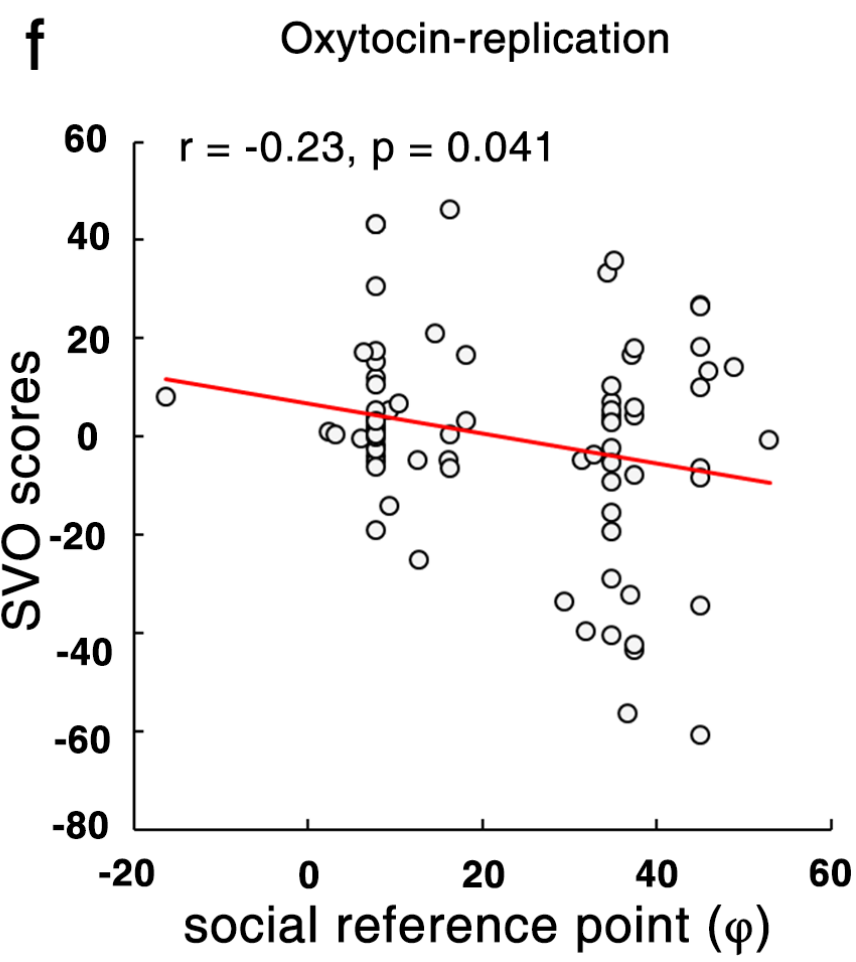


a

Deviation from individual-specific reference-point

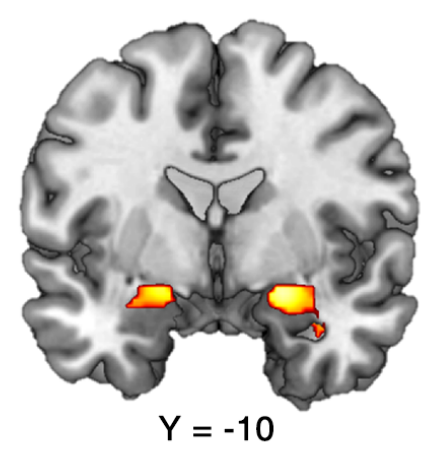

3.1 5.0

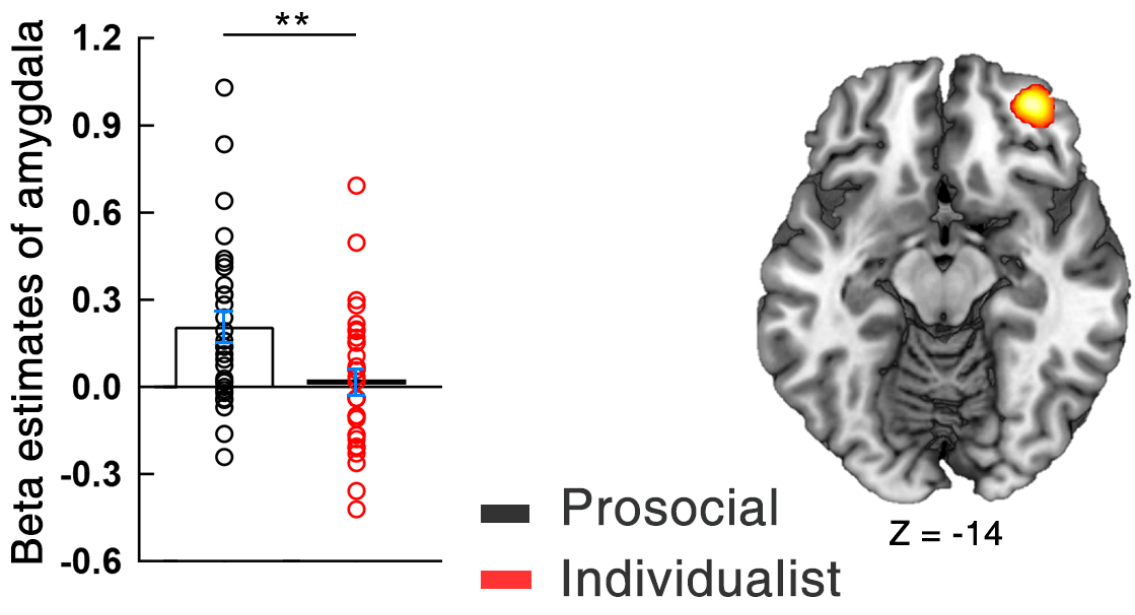

b Deviation from individual-specific reference-point

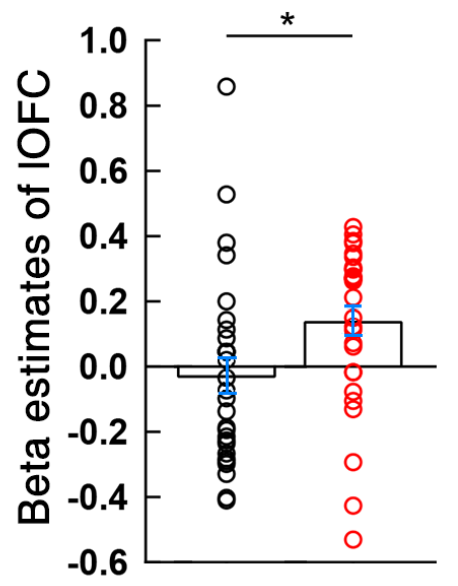


a

Deviation from individual-specific reference-point

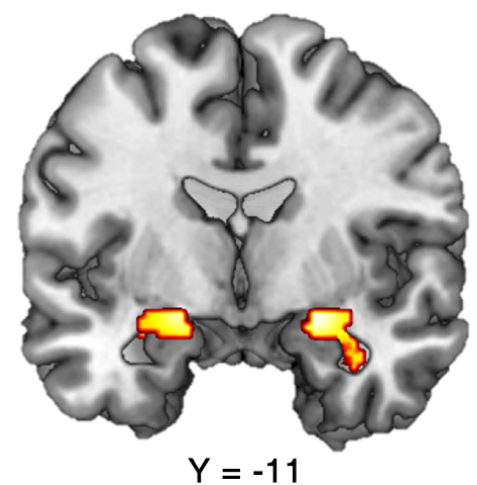

t-value
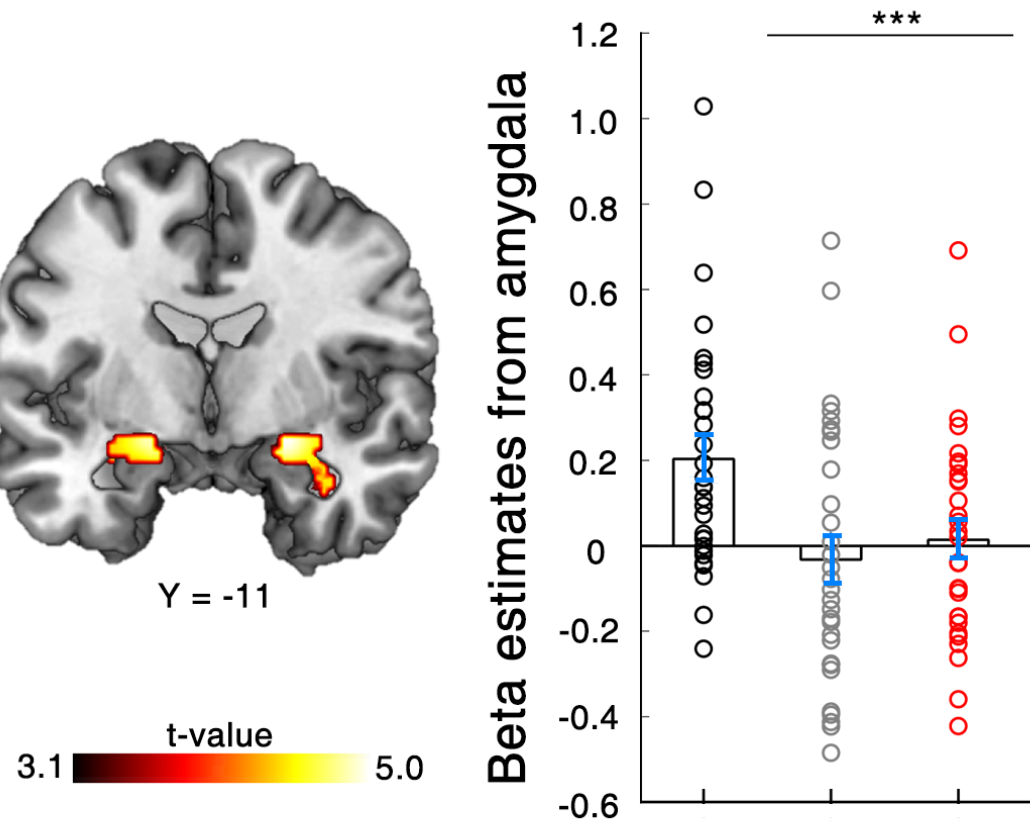

5.0

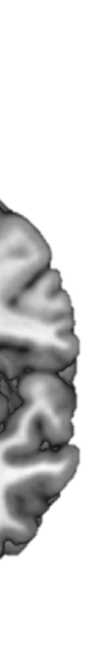

b

Amygdala prediction of trial-by-trial preference

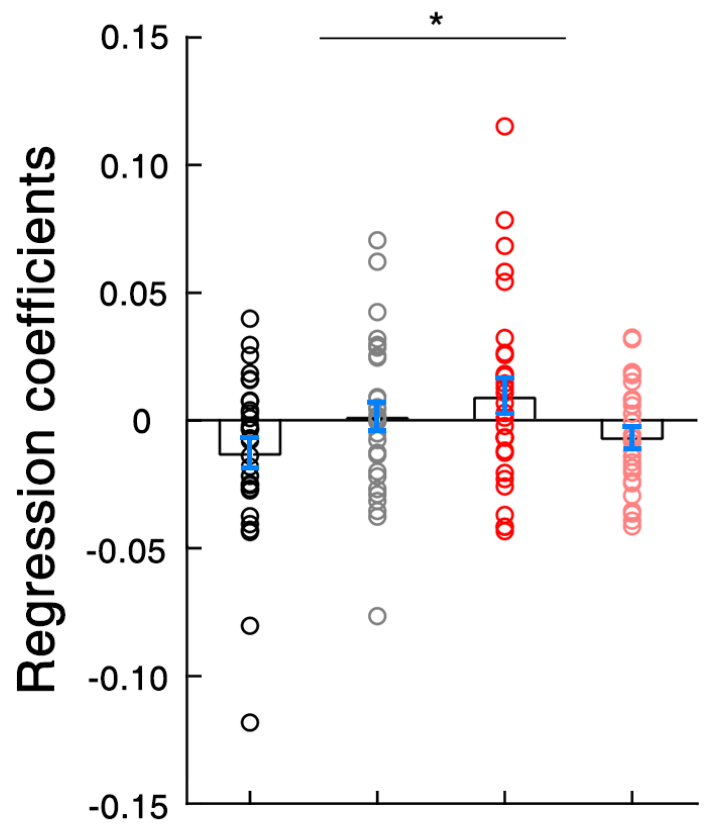

